\title{
CHARGE COLLECTION MECHANISMS IN AIGaN/GaN MOS HIGH ELECTRON MOBILITY TRANSISTORS
}

\author{
By \\ Isaak Knox Samsel \\ Thesis \\ Submitted to the Faculty of the \\ Graduate School of Vanderbilt University \\ in partial fulfillment of the requirements \\ for the degree of \\ MASTER OF SCIENCE \\ in \\ ELECTRICAL ENGINEERING
}

August, 2014

Nashville, Tennessee

Approved:

Professor Robert A. Reed

Professor Ronald D. Schrimpf 


\section{ACKNOWLEDGMENTS}

For this work, I must begin by expressing my sincere thanks to all those who guided my academic growth and my research pursuits. Principally, I must express my gratitude toward my academic advisor, Professor Robert Reed, as well as many members of the Electrical Engineering faculty in the Radiation Effects and Reliability group of Vanderbilt University including Professors Ron Schrimpf, Dan Fleetwood, and Robert Weller. A special and specific thanks must additionally be given to Professor Enxia Zhang, without whom none of the experimental work contained herein would have been possible.

I would also like to thank each of the co-authors who appear on the IEEE TNS paper sharing the same title as this composition: Nick Hooten and Geoff Bennett of Vanderbilt University provided guidance and computer code for processing my experimental data; Erik Funkhouser and Mike McCurdy of Vanderbilt University, as well as George Vizkelethy of Sandia National Laboratories were also instrumental in performing experimental work; Xiao Sun and T. P. Ma provided experimental devices to me and our research group; Omair Saadat and Tomás Palacios fabricated the experimental devices on which this work is based. Thanks is also due to Dennis "Scooter" Ball, for his knowledge of TCAD simulation software.

Personal thanks are due to my friends and family. Firstly, I thank my mother and father, who have always instilled in me a scientific mindset and nurtured my curiosity. I must also thank my friends from the University of Tennessee, Eric Martin and Joe Carboni, each of the three of us having decided to pursue graduate degrees in our respective fields (it seemed like a good idea at the time). We have all remained supportive of one another through good-natured needling and ego-pruning. I also thank my friends at Vanderbilt University, especially the ones who are forced to share an office

with me; I will refrain from constructing a list out of simple fear that I will leave someone out. Last but certainly not least, very special thanks is also due to my fiancée, Kayla Shupe for her unending support and patience.

Financial support for this work was provided in part by the American taxpayer, through the Defense Threat Reduction Agency, as well as the Air Force Research Laboratory. 


\section{TABLE OF CONTENTS}

ACKNOWLEDGMENTS $\ldots \ldots \ldots \ldots \ldots \ldots \ldots \ldots \ldots \ldots \ldots$

LIST OF FIGURES $\ldots \ldots \ldots \ldots \ldots \ldots \ldots \ldots \ldots \ldots$

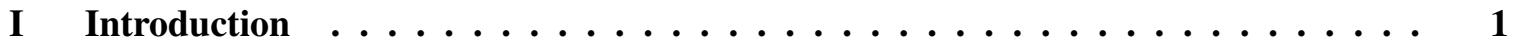

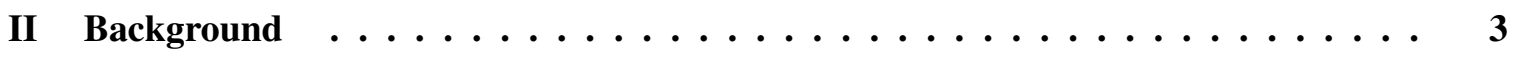

II.1 Gallium Nitride and GaN-Based Devices . . . . . . . . . . . . . . . . . . . . 3

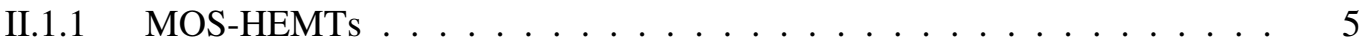

II.2 The Space Radiation Environment . . . . . . . . . . . . . . . . . . . . . . 7

II.2.1 Trapped Radiation Environments . . . . . . . . . . . . . . . . . . 7

II.2.2 Galactic Cosmic Rays . . . . . . . . . . . . . . . . . . . . 8

II.3 Singe-Event Effects in Microelectronics _ . . . . . . . . . . . . . . . . . 10

II.3.1 Single-Event Charge Collection . . . . . . . . . . . . . . . . . . . . . . . 12

II.3.2 Single-Event Gate Rupture . . . . . . . . . . . . . . . . . . . . . . . . . . 13

II.3.3 Transient Response Measurement . . . . . . . . . . . . . . . . . . . . . . 14

II.4 Previous Work . . . . . . . . . . . . . . . . . . . . . . . 16

II.4.1 SEEs in Other Material systems _ . . . . . . . . . . . . . . . . 16

II.4.2 AlGaN/GaN HEMTs . . . . . . . . . . . . . . . . . . . . . . 24

III Experimental Methodology $\ldots \ldots \ldots \ldots \ldots \ldots$

III.1 Device Description _ . . . . . . . . . . . . . . . . . . . . . . . 26

III.2 Pulse Capture Setup . . . . . . . . . . . . . . . . . . . . 26

IV Charge Collection Measurements . . . . . . . . . . . . . . . 31

IV.1 Broadbeam Irradiation . . . . . . . . . . . . . . . . . . . . . . . . 31

IV.1.1 Experimental Setup . . . . . . . . . . . . . . . . . . . 31

IV.1.2 Results . . . . . . . . . . . . . . . . . . . 32

IV.2 Microbeam Irradiation . . . . . . . . . . . . . . . . . . . . 33

IV.2.1 Experimental Setup . . . . . . . . . . . . . . . . 33

IV.2.2 Results . . . . . . . . . . . . . . . . . . . 33

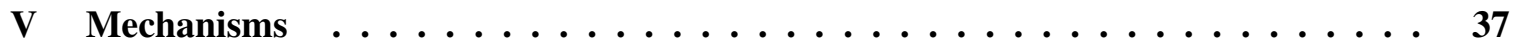

V.1 Simulation Setup . . . . . . . . . . . . . . . . . . . . . . 37

V.2 Simulation Results . . . . . . . . . . . . . . . . . . . 37

V.3 Discussion ............................ 40

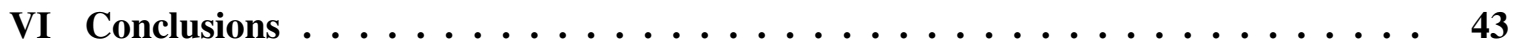


BIBLIOGRAPHY $\ldots \ldots \ldots \ldots \ldots \ldots \ldots \ldots \ldots \ldots \ldots \ldots \ldots$ 


\section{LIST OF FIGURES}

Figure Page

II.1 (a) Diamond cubic unit cell. (b) Hexagonal structure of wurtzite crystal lattice.

(c) Unit cell of the wurtzite crystal structure . . . . . . . . . . . . . . . 4

II.2 (a) $\mathrm{I}_{O n} / \mathrm{I}_{O f f}$ ratios for $\mathrm{HfO}_{2^{-}}, \mathrm{Al}_{2} \mathrm{O}_{3^{-}}$, and Schottky-gate GaN HEMT devices over a range of oxide deposition temperature. Note the significant improvement over Schottky-gate devices, nearing four orders of magnitude in some devices.(b) $\mathrm{I}_{O n} / \mathrm{I}_{O f f}$ ratios for similar transistors over a range of anneal temperatures. . . . .

II.3 Graphic qualitatively depicting the belt-like nature of the near-Earth trapped radiation environment, i.e., the Van Allen belts. The "Horns" refer to the highaltitude protrusions of the outer belt. . . . . . . . . . . . . . . .

II.4 Cosmic ray flux per solid angle for ions with atomic number 1 through 92 in GEO at solar minimum behind 100 mils aluminum shielding. Note the relative abundance of atomic species. . . . . . . . . . . . . .

II.5 Integral iron LET spectrum and total galactic cosmic ray (GCR) LET spectrum of the cosmic ray environment. LET in this range is generally considered most significant in terms of single-event effects. . . . . . . . . . .

II.6 LET Spectra of heavy ions at LEO $(500 \mathrm{~km})$ at various inclinations. Note that geomagnetic shielding is most significant at the equator (0 degrees inclination). .

II.7 Simple illustration of the charge generation process. Left to right across the figure: an incident ion deposits energy; the energy liberates charge (electronhole pairs); some charges move to the device terminal; collected charge appears as transient current. . . . . . . . . . . . . . . .

II.8 Simple illustration of the pileup of holes near gate dielectric along the path of an ion strike. Holes are swept up toward the oxide, where the momentarily collect before diffusing laterally to the source. . . . . . . . . . . .

II.9 Block diagram of an example time-resolved charge collection measurement system. Looking from the DUT, the bias tee decouples the AC transient signal from the DC bias.

II.10 Ion-induced current transients in InAlAs/InGaAs HEMTs, plotted on three different timescales. Note the change in dominant contributing mechanism at different times. . . . . . . . . . . . . . . . 
II.11 Illustration of the so-called "field funneling effect." (a) An alpha particle strikes a pn-junction, then (b) the depletion region collapses, and (c) the potential is modulated along the particle track. . . . . . . . . . . . . .

II.12 Collected charge measurements in semiconducting and semi-insulating GaAs. The higher collected charge in SC GaAs is attributed to the greater contribution of "field funneling" in the semiconducting substrate. . . . . . . . . . .

II.13 Vertical cross-section equilibrium band diagram of InAlSb/InAs/AlGaSb HEMT. 20

II.14 Barrier lowering pre- and post strike in threshold and accumulation, obtained from a horizontal cutline through the center of the channel. . . . . . . . . . .

II.15 Current transients observed during ion strikes in SiC MESFETs, with device biased in ON-state. Strike location is (a) in between gate and source, (b) at the gate, (c) in between gate and drain, (d) at the drain. . . . . . . . . . . . .

II.16 The bond pads on this SiC Schottky barrier diode, and the burnout traces circled, show the material destruction caused by neutron-induced SEB. . . . . . . . .

II.17 Gate current in GaN HEMTs under $236 \mathrm{MeV}$ Br irradiation. Note that device under lower $-5 \mathrm{~V}$ gate bias demonstrated little change in gate current, while the $-15 \mathrm{~V}$ bias condition produced both "soft" degradation in gate current, as well as total failure. . . . . . . . . . . . . . . . .

III.1 (a) Schematic cross-section of AlGaN/GaN MOS-HEMT. (b) Typical band diagram of $\mathrm{AlGaN} / \mathrm{GaN}$ interface showing 2DEG. . . . . . . . . . . . . . .

III.2 DC characteristics of $\mathrm{HfO}_{2}$-gate DUT. Drain Current is measured as a function of gate voltage. Current measurements are shown for three drain voltage conditions.

III.3 Layout mask of AlGaN/GaN DUT. Two devices share a gate and drain, while having separate source contacts. . . . . . . . . . . . . . .

III.4 Custom-milled high-speed package fabricated at Vanderbilt University specifically for time-resolved charge collection measurements. Note the microwave transmission lines carrying the signal to the bulkheads. . . . . . . . . . .

III.5 S-parameters of custom-made high-speed package as a function of frequency up to $50 \mathrm{GHz}$. $S_{11}$ tracks $S_{22}$ almost exactly, and $S_{12}$ tracks $S_{21}$ almost exactly. The insert figure is the TDR response of the same package. . . . . . . . . . .

IV.1 Drain and gate current transients produced in the DUT by $14.3 \mathrm{MeV}$ oxygen ions: $\mathrm{V}_{G E}=-0.6 \mathrm{~V}, \mathrm{~V}_{D}=500 \mathrm{mV}, \mathrm{V}_{S A}=0 \mathrm{~V}, \mathrm{~V}_{S B}=0 \mathrm{~V}$. Note the symmetry of the gate and drain signals, and the absence of any transient signal on the source channels. . . . . . . . . . . . . . . . . . 
IV.2 (a) $10 \mu \mathrm{m} \times 10 \mu \mathrm{m}$ area scan of a Schottky-gate device. (b) Comparable area scan of an $\mathrm{HfO}_{2}$-gate device. Each point on each plot represents a detected transient event (gate-channel scope trigger). Note the strong positional dependence on strike location. The number of observed transient events is almost two orders of magnitude greater in the Schottky-gate device compared to the $\mathrm{HfO}_{2}$ gate oxide device. . . . . . . . . . . . . . . . . . . . . .

IV.3 Mean collected charge in identical $10 \mu \mathrm{m} \times 10 \mu \mathrm{m}$ area scans of a $4 \mu \mathrm{m}$ gate Schottky-gate device. Total collected gate charge trends weakly with increasingly negative gate bias. . . . . . . . . . . . . . . . .

V.1 Simulated device structure showing relevant dimensions and a particular strike location. The resulting gate current transient from such a strike simulation is shown in the inset. . . . . . . . . . . . . . . .

V.2 Equilibrium band diagram of on $\mathrm{HfO}_{2}$-gate device. The oxide layer introduces a slight valence band barrier between the gate and $\mathrm{AlGaN}$ layer. . . . . . . . . . .

V.3 Equilibrium band diagram of Schottky-gate device. The absence of a gate oxide layer in these devices promotes hole collection at the gate. This is due to the lack of a valence band barrier between the gate metal and the semiconductor material.

V.4 Equilibrium band diagram of $\mathrm{Al}_{2} \mathrm{O}_{3}$-gate device. The oxide layer introduces significant valence band barrier relative to the barrier in the $\mathrm{HfO}_{2}$-gate device. .

V.5 Direct comparison of typical gate transient currents in $\mathrm{HfO}_{2}$-gate (top) and Schottkygate (bottom) devices. Note the larger peak current in the Schottky-gate device transient. . . . . . . . . . . . . . . . .

V.6 Conduction band energy along a horizontal cutline following the channel of a Schottky-gate device in the source-to-drain direction. The far left and far right regions of the figure are the source-gate and gate-drain separation regions respectively. The strike induces localized conduction band distortion, while the band energy on the side opposite the strike remains largely unchanged. This helps explain the reliable isolation of events in the source or drain as shown in Fig. IV.2.

V.7 Magnitude of the electric field along vertical cutlines through the gate of a Schottkygate device, focused at the top of the GaN layer. The electric field has peaks near the edges of the gate stack, where the increased strength and depth of the field increase the transient current response in those regions. . . . . . . . . . 


\section{CHAPTER I}

\section{Introduction}

An ever-growing number of alternative semiconducting materials have been investigated for applications in all areas of microelectronics. Naturally, devices fabricated using materials other than silicon have also been investigated for applications in space, and other environments in which ionizing radiation is a serious reliability concern. Amongst the reliability concerns in these environments, single-event effects pose risks ranging from logic errors (single-event upset), to catastrophic failure and the destruction of parts (single-event latchup, single-event gate rupture, single-event burnout, etc.). Since each of these effects is necessarily initiated by the generation and collection of charge in the device, study of the underlying mechanisms behind charge collection, often through the measurement of radiation-induced single-event transients, has proven to provide useful insight into various single-event effects, and led to methods of mitigation [1].

The fast operating speeds of III-V compound semiconductor devices make them attractive alternatives to silicon-channel MOSFETs in applications where high-power and high-speed performance are imperatives. For high-speed technologies fielded in harsh radiation environments (e.g., telecommunications satellites and other space-based applications) gallium nitride $(\mathrm{GaN})$ is more robust to radiation damage than many other III-V materials [2-5]. Further, the wide bandgap, along with other material properties, afford GaN impressive applicability in other extreme environments such as those at high temperatures. A short list of some of these parameters, including electron mobility $(\mu)$, relative permittivity $(\varepsilon)$, bandgap $\left(\mathrm{E}_{g}\right)$, and maximum operating temperature $\left(\mathrm{T}_{\text {Max }}\right)$, are compared with those of other familiar semiconductors in Table I.1.

\begin{tabular}{|c|c|c|c|c|}
\hline Material & $\mu\left(\mathrm{cm}^{2} / \mathrm{V} \cdot \mathrm{s}\right)$ & $\varepsilon$ & $\mathbf{E}_{g}(\mathrm{eV})$ & $\mathbf{T}_{\operatorname{Max}}\left({ }^{\circ} \mathrm{C}\right)$ \\
\hline Si & 1300 & 11.4 & 1.1 & 300 \\
\hline GaAs & 5000 & 13.1 & 1.4 & 300 \\
\hline SiC & 260 & 9.7 & 2.9 & 600 \\
\hline GaN & 1500 & 9.5 & 3.4 & 700 \\
\hline
\end{tabular}

Table I.1: A few physical parameters of common semiconducting materials.

Oxide-insulated $\mathrm{AlGaN} / \mathrm{GaN}$ high electron mobility transistors (HEMTs) exhibit reduced gate 
current under normal operating conditions, and exhibit on/off current ratios improved by several orders of magnitude, as compared to equivalent Schottky-gate devices [6-8]. There is limited singleevent-effect (SEE) information on AlGaN/GaN HEMT devices [9, 10]. Single event gate rupture was evaluated for AlGaN/GaN HEMTs in [10].

Experimental work in this thesis reports the results of ion-induced charge-collection experiments in $\mathrm{AlGaN} / \mathrm{GaN}$ metal-oxide-semiconductor (MOS) HEMTs and Schottky-gate HEMTs. Results were obtained using broadbeam 14.3 MeV oxygen ions and microbeam $48 \mathrm{MeV}$ silicon ions. In each case, the transient signals were captured in real time. For the microbeam data, the location of individual ion strikes was also recorded. Depending on strike location, current transients appear either between the gate and the drain, or between the gate and the source. Analysis of the band structure of the device using 2D TCAD simulations shows that hole collection at the gate is favorable under off-state conditions, even for devices with an insulating $\mathrm{HfO}_{2}$ oxide layer. In contrast, the valence band barrier created by an $\mathrm{Al}_{2} \mathrm{O}_{3}$ gate-oxide reduces hole collection to a level below the resolution of the test setup. 


\section{CHAPTER II}

\section{Background}

\section{II.1 Gallium Nitride and GaN-Based Devices}

The physical mechanisms behind the operation of GaN-based semiconductor devices are fundamentally different than those that govern silicon-based devices. Contrary to the (relatively) familiar behavior of doped silicon $\mathrm{p}-\mathrm{n}$ junctions that underlie the operation of bipolar junction transistors (BJTs) and metal-oxide-semiconductor field effect transistors (MOSFETs), entirely separate physical phenomena are responsible for the operation of GaN devices of interest such as HEMTs. While a thorough exploration of the physics of GaN HEMTs (or HEMTs in general, for that matter) could fill volumes, a few key points will be discussed in this section, with emphasis placed on the deviation from more familiar aspects of device physics that govern silicon MOSFETs.

The differences between GaN and silicon begin at the level of atomic crystalline structure. Device physics students will inevitable become intimately familiar with the crystal structure exhibited by electronics-grade silicon: the diamond lattice. In contrast, GaN (as well as many other IIIV semiconducting materials) exhibits the wurtzite crystal structure. GaN can also be grown in a zincblende crystallographic configuration, but these crystals are less stable, and the wurtzite polytype is both more common and more relevant to electronics applications [11]. The wurtzite crystal structure is illustrated in Fig. II.1, along with the more-familiar diamond crystal structure.

As made clear by comparing their respective unit cells, the wurtzite structure is lacking in symmetry when compared to the diamond lattice. This lack of so-called inversion symmetry inherent in wurtzite $\mathrm{GaN}$ plays a fundamental role in polarization, which is intimately linked with the material's bandstructure and the free charge carriers that will govern the behavior of an electronic device. In materials with higher symmetry, mechanical strain (such as that resulting from the growth of a thin film of material on a substrate with a mis-matched lattice constant) is the dominant contributor to this polarization, which represents the physical mechanism underlying the operation of familiar devices such as crystal oscillators: the piezoelectric effect. However, a generalized formula to quantify polarization would follow the form: 


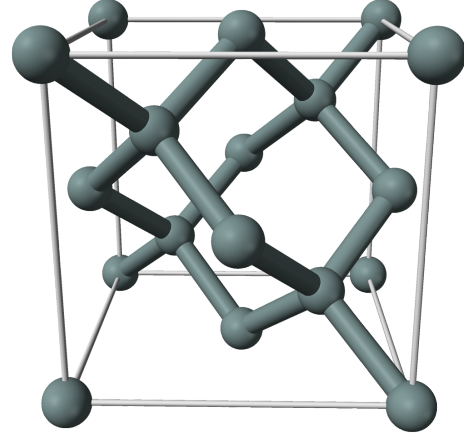

(a) Diamond Cubic

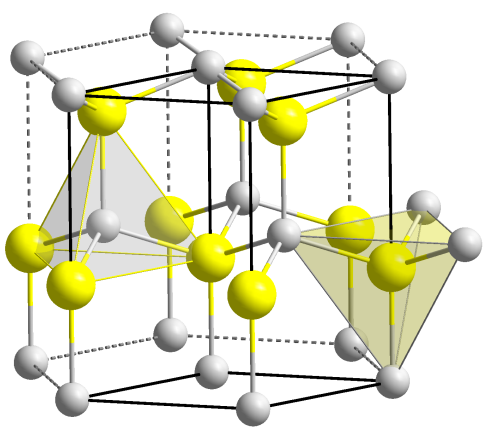

(b) Wurtzite Structure

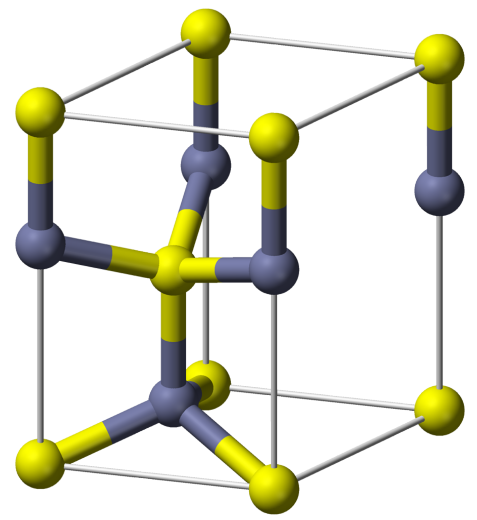

(c) Wurtzite Unit Cell

Figure II.1: (a) Diamond cubic unit cell. (b) Hexagonal structure of wurtzite crystal lattice. (c) Unit cell of the wurtzite crystal structure.

$$
P_{\text {Total }}=\Delta P_{S p}+P_{P E}
$$

Where $P_{P E}$ refers to the piezoelectric component of the polarization density induced by some mechanical strain. The units are equivalent to an areal charge density $\left(C / m^{2}\right)$. The $P_{S p}$ term refers to the spontaneous polarization which would be present even in a state of zero strain, and is a fundamental physical parameter of a given material system. The $\Delta$ in the spontaneous polarization term is present because this equation specifically applies not to homogeneous bulk material, but to a material interface; thus, $\Delta P_{S p}$ actually refers to a difference in spontaneous polarization across a heterointerface [12].

Just as the p-n junction is often a locus of physical understanding in terms of the operation of a device such as a silicon BJT, heterostructures often play the same role in understanding the operation of HEMTs. In GaN-based technology, the most common heterostructure is the material interface between $\mathrm{GaN}$ and $\mathrm{AlGaN}$. $\mathrm{AlGaN}$ is a ternary compound, and can be grown with a range of different aluminum concentrations, so the chemical formula could be written less ambiguously as $\mathrm{Al}_{x} \mathrm{Ga}_{1-x} \mathrm{~N}$, where $x$ is commonly called the mole fraction. Note that a mole fraction $x=0$ would simply represent $\mathrm{GaN}$, and a mole fraction $x=1$ would represent aluminum nitride (AlN). The mole fraction defines important properties of the heterostructure, such as the spontaneous polarization 
mentioned previously.

The (properly controlled) heterointerface between $\mathrm{AlGaN}$ and $\mathrm{GaN}$ produces an interesting and useful result: a two-dimensional electron gas (2DEG). An electric displacement field $\mathbf{D}$ is defined by the equation:

$$
\mathbf{D}=\varepsilon \mathbf{E}+\mathbf{P}
$$

Where $\varepsilon \mathbf{E}$ represents a screening field caused by free carriers. Therefore, in an ideally undoped material system, the equation reduced to $\mathbf{D}=\mathbf{P}$. In other words, the polarization density establishes the electric field responsible for the band structure of the $\mathrm{AlGaN} / \mathrm{GaN}$ heterointerface that tightly confines electrons in a 2DEG.

Transistors built using the $\mathrm{AlGaN} / \mathrm{GaN}$ heterostructure are appropriately called high electron mobility transistors because they are able to take advantage of the exceptional electron mobility of GaN (See Table I.1). Typically, ohmic contacts are formed by depositing a metal (stacks such as $\mathrm{Ti} / \mathrm{Al} / \mathrm{Ni} / \mathrm{Au}$ are common [13]) and annealing at high temperatures to form the source and drain. Gate stack fabrication varies more widely, and is still a subject of research [14].

\section{II.1.1 MOS-HEMTs}

Currently, GaN-based electronic devices such as GaN HEMTs (for the sake of brevity, from this point on unless otherwise stated, "GaN HEMTs" or "GaN devices" refer to devices built upon the $\mathrm{AlGaN} / \mathrm{GaN}$ heterointerface) already exist as commercial, off-the-shelf (COTS) parts. Commercially available parts are generally utilized for high-power RF applications, and are generally based on relatively simple HEMT architecture. However, a tremendous amount of research and consequent physical understanding sits at the foundation of even the most simple GaN HEMT architecture, and the research that has gone into fabrication methods alone is quite substantial [13, 14].

Such research has proven fruitful, and since the first GaN HEMTs were first reported 1993 [15], operation has been improved substantially on a number of fronts [13]. A fairly intuitive improvement to the HEMT architecture, though one that has proven difficult to implement, is the addition of an insulating oxide layer between the gate and the device terminal, improving, among other parameters, gate leakage current. Given the common high-power application of GaN HEMTs, where 


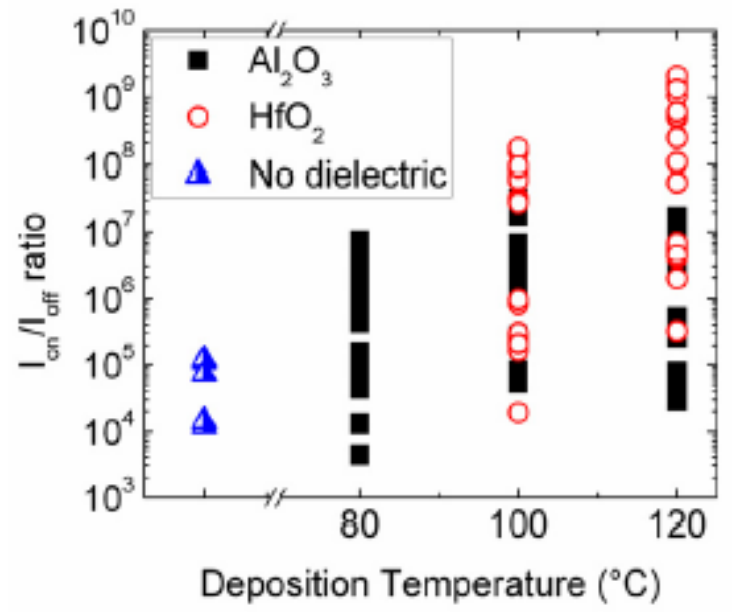

(a)

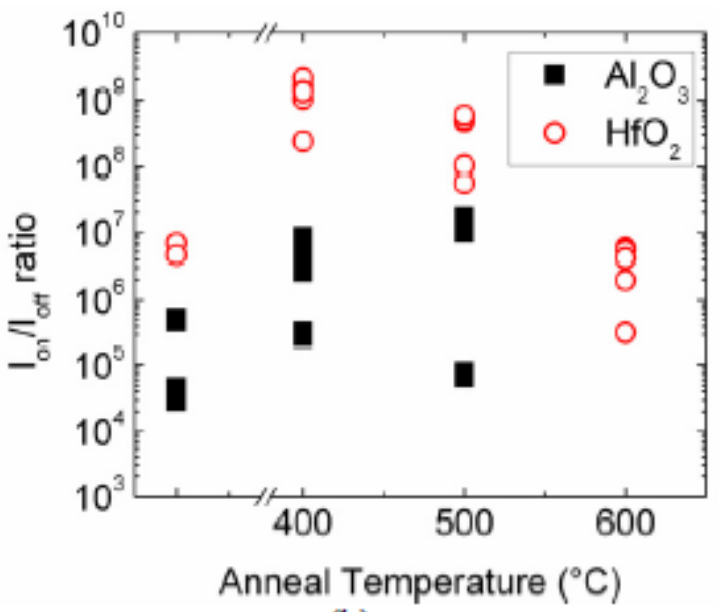

(b)

Figure II.2: (a) $\mathrm{I}_{O n} / \mathrm{I}_{O f f}$ ratios for $\mathrm{HfO}_{2^{-}}, \mathrm{Al}_{2} \mathrm{O}_{3^{-}}$, and Schottky-gate GaN HEMT devices over a range of oxide deposition temperature. Note the significant improvement over Schottky-gate devices, nearing four orders of magnitude in some devices.(b) $\mathrm{I}_{O n} / \mathrm{I}_{O f f}$ ratios for similar transistors over a range of anneal temperatures. After [6].

operating voltages commonly exceed $20 \mathrm{~V}$, excessive gate leakage current can be a nuisance for device performance. Such gate leakage contributes to degradation in breakdown voltage, power-added efficiency, and contributes to noise [16].

Earlier investigations of gate dielectrics included $\mathrm{SiO}_{2}, \mathrm{Si}_{3} \mathrm{~N}_{4}, \mathrm{Al}_{2} \mathrm{O}_{3}$, and others, but the processes used in forming these dielectrics suffer from unrealistic complexity and limited scalability when viewed from the perspective of large-scale manufacturing. Atomic layer deposition (ALD), where materials are chemically deposited one atomic-layer at a time, has been demonstrated as a method for growing oxides that is not only superior to previous methods in terms of scalability, but that also produces $\mathrm{Al}_{2} \mathrm{O}_{3}$ that is of much higher quality (in terms of defect density, stoichiometry, etc.) than previously used methods [16].

ALD processes have been utilized to fabricate devices with additional high-k materials, such as $\mathrm{HfO}_{2}$. In addition to gate leakage current, insulating gate oxides also improve the on/off current, thereby reducing power loss, especially for high-speed switching applications. Fig. II.2 shows how significant this improvement can be relative to otherwise comparable Schottky-gate HEMT devices. Note that the same fabrication method reported in [6] was used to produce all of the experimental devices detailed in subsequent sections. 


\section{II.2 The Space Radiation Environment}

Device applications that subject electronic components to non-terrestrial environments must carefully consider the ionizing radiation characteristics of such environments. Serious concern for the radiation environment in space and corresponding quantitative measurements date back to the infancy of space exploration in the 1950s. The first American satellite, Explorer I, was launched into orbit in January of 1958 equipped with instruments thought capable of quantifying the predicted near-Earth radiation environment. The failure of this instrument due to the overwhelming intensity of the radiation environment it was subjected to provided the first glimpse into the challenges associated with quantifying and mitigating the effects of such environments [17].

Essentially, the radiation environment of outer space is divided into two categories: trapped particles and transient phenomena [17]. Trapped, charged particles are held near the Earth by its magnetosphere (a phenomenon present in some other planetary systems, as well). These consist primarily of protons and electrons, but can also include heavier ions. Transient phenomena includes energetic particles of solar origin, but also includes galactic cosmic rays (GCRs) that can be made up of any element from the periodic table. Given the drastically different nature of these populations in terms of origin, species, and energy, they each introduce unique challenges to effective mitigation.

\section{II.2.1 Trapped Radiation Environments}

The prediction that a planetary magnetosphere could be capable of trapping ionizing radiation was made as early as the 19th century. Norwegian physicist Kristian Birkeland first proposed the idea based on his observation that charged particles spiraled around magnetic field lines in 1895 . Despite this early prediction, however, a serious effort to quantitatively model the near-Earth trapped radiation environment was not undertaken for more than a half-century [17].

John Van Allen is generally credited with the confirmation of the existence of the trapped electron and proton environment surrounding the Earth, based on measurements from the first satellites launched by the Explorer program. An artist's depiction of the appropriately-named "Van Allen radiation belts" is shown in Fig. II.3, which illustrates the lower-flux "slot" region between inner and outer belts. Though measurements of this radiation environment continued persistently, the results differed by as much as an order of magnitude, for reasons that were, at the time, elusive. The motivation for a quantitative model of the trapped radiation environment came through the eventual 


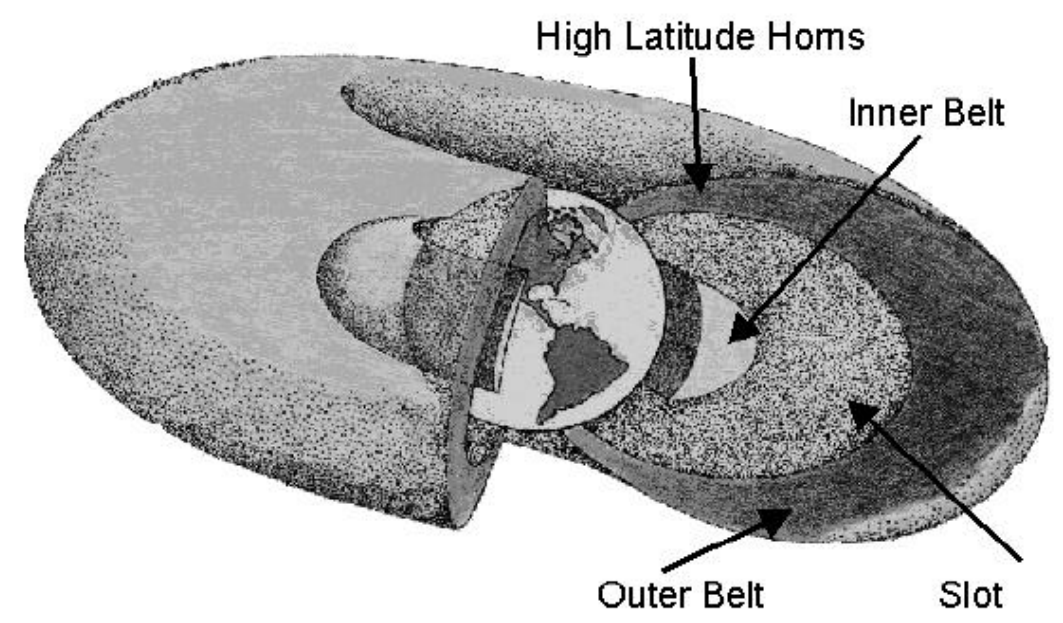

Figure II.3: Graphic qualitatively depicting the belt-like nature of the near-Earth trapped radiation environment, i.e., the Van Allen belts. The "Horns" refer to the high-altitude protrusions of the outer belt. After [18].

failure of spacecraft electronics, exacerbated greatly by the high-altitude nuclear testing of the early 1960s, the radioactive signatures of which dominated the trapped radiation environment for years afterward. A more thorough history of the development of such models is discussed in [17].

\section{II.2.2 Galactic Cosmic Rays}

Cosmic rays are particles with energies covering a vast spectrum that are present primarily beyond the protection of the Earth's magnetosphere and atmosphere (though some lighter particles may penetrate to the planet's terrestrial environment). They are of significant concern for single-event effects in microelectronics, as particles of sufficient energy and mass can deposit an enormous amount of energy in sensitive components.

The first experimental observations of cosmic rays resulted from the erroneous conclusion of early twentieth-century scientists that sensitive experiments were being subjected to radiation coming from contaminated materials and the Earth itself. Doubt was cast on this hypothesis after Nobel laureate Victor Hess performed a somewhat whimsical experiment in which he measured incident radiation while ascending in a hot air balloon. Contrary to expectation, radiation increased with altitude, thereby identifying the source of the troublesome radiation as outer space. The term "cosmic rays" was coined by Robert Millikan after performing subsequent experiments to confirm Hess's 
conclusions [17].

Interest in modeling the cosmic ray environment was slower in gaining momentum than for trapped radiation. Cosmic rays were reasonably thought to have a relatively insignificant contribution to problems such as total ionizing dose, and thus for nearly two decades into the space program were not thought to be of real concern for electronics in space. The real concern for the cosmic ray environment, single-event effects, weren't even proven to exist until the mid-1970s, as discussed further in the next section. This history is elaborated in [17].

Cosmic rays capable of inducing problematic single-event effects include single protons, as well as all heavier ions (atomic nuclei of any species of atomic number greater than 1, without their electron shells). The mass distribution predictably corresponds to the natural relative abundances of the elements, but can be substantially altered by solar cycle. Cosmic rays originating outside the solar system, which include the heaviest and most energetic particles, are isotropic, that is, a given point in space is subject to cosmic rays from all directions. Cosmic ray flux can therefore be given in units of particles $/ \mathrm{cm}^{2}$, where the area refers to the area of a sphere through which cosmic rays pass along arbitrary chord lengths, i.e., not necessarily through the center of the sphere [19].

Models of the cosmic ray environment have naturally been revised and updated as the amount of experimental data measuring cosmic rays has grown, as have the methods for predicting the effect of the environment on microelectronics. Of particular note is the Cosmic Ray Effects on MicroElectronics (CREME, or more recently, CRÉME) family of code [20]. The most recent release of this code utilizes an international standard model of the galactic cosmic ray environment [21] along with radiation transport code to predict how cosmic rays will affect a device that is defined by the user. A cosmic ray kinetic energy spectrum produced by CRÉME is shown in Fig. II.4.

As discussed in the next section in more detail, the linear energy transfer (LET) of a particle is an extremely important metric used in predicting how heavy ions will affect vulnerable microelectronics. For this reason, LET spectra are somewhat more instructive than energy spectra with regard to evaluating how an environment will affect electronic devices. An integral LET spectrum produced using CREME96 is presented in Fig. II.5. Note that in this figure, the contribution of iron ions to the total is plotted separately, since iron's abundance and range of LET makes it significant to electronic devices in this environment.

Just as the Earth's magnetic field created the trapped radiation environment, it also complicates 


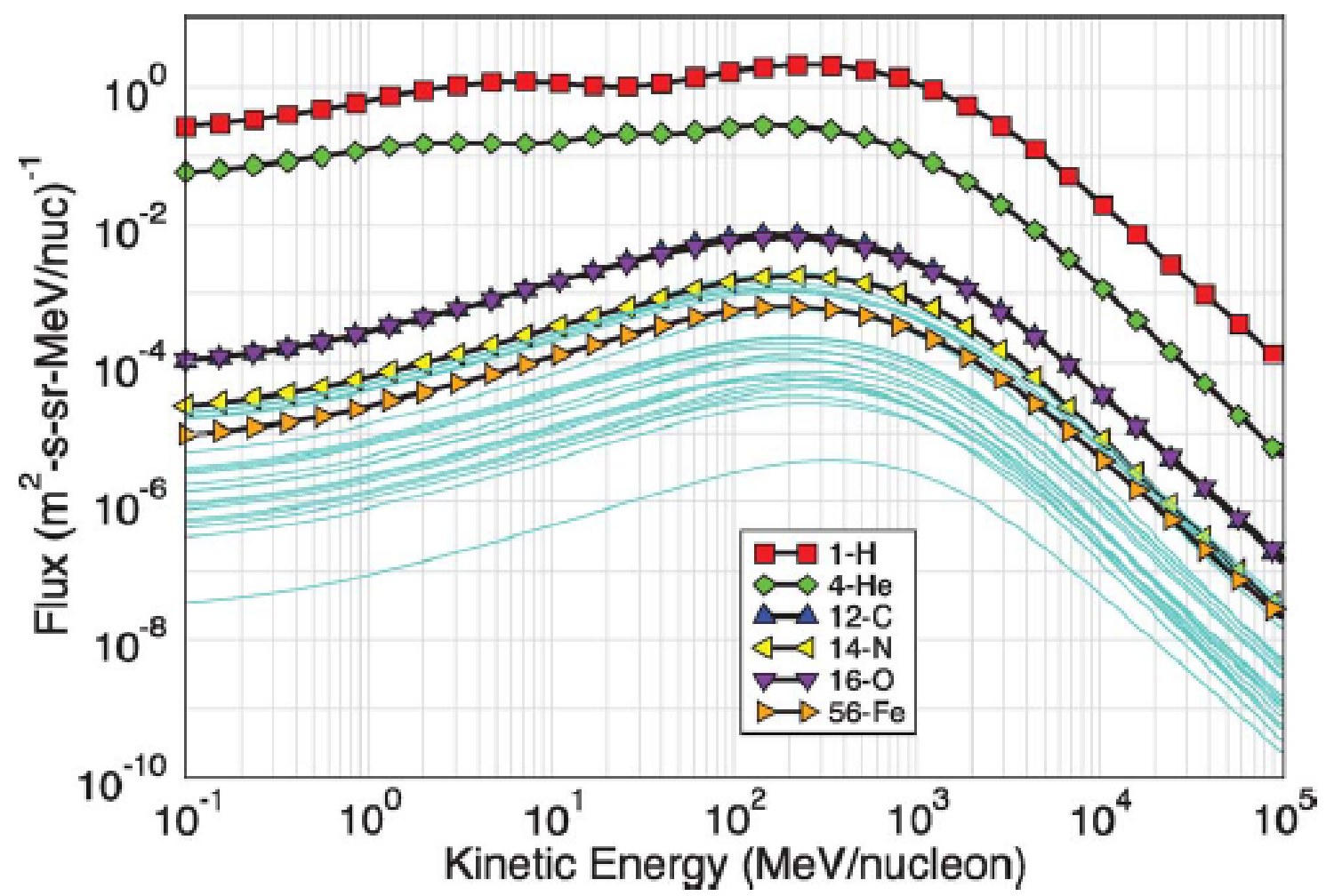

Figure II.4: Cosmic ray flux per solid angle for ions with atomic number 1 through 92 in GEO at solar minimum behind 100 mils aluminum shielding. Note the relative abundance of atomic species. After [20].

the cosmic ray environment near the planet. The magnetosphere serves as a geomagnetic shield, which can reject some of the incident charged particles. As implied by figure II.3, the Earth's magnetic field interacts most strongly with charged particles near the equator, the magnetic field near the equator therefore produces the most significant shielding effect. An example of how this shielding effect changes the LET spectrum is shown in Fig. II.6. The figure shows that the LET spectrum is most severely diminished at an inclination of 0 degrees, showing the most significant geomagnetic shielding taking place at the equator.

\section{II.3 Singe-Event Effects in Microelectronics}

Single-event effects (SEEs) are a broad class of semiconductor device or integrated circuit malfunctions that are each initiated by a single incident energetic particle. Regardless of device or circuit, each SEE shares a common genesis: the generation and subsequent collection of charge induced by an energetic particle. As an incident particle passes through the device material, it loses energy by 


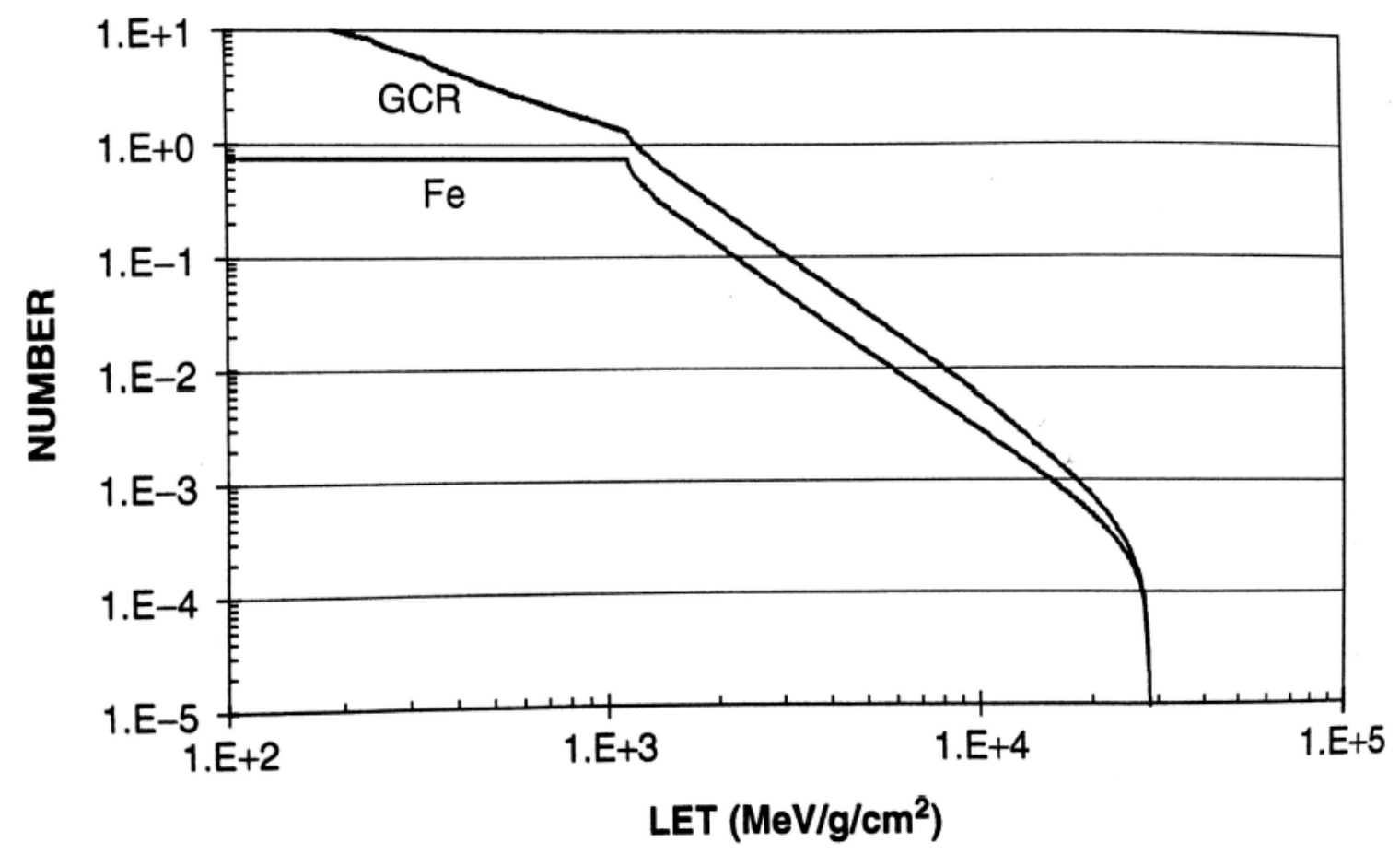

Figure II.5: Integral iron LET spectrum and total LET spectrum of cosmic ray environment. LET in this range is generally considered most significant in terms of single-event effects. After [19].

interacting with it. Through either direct or indirect ionization, incident energetic particles will lose their energy by liberating electron-hole pairs along their path.

Single-event upsets (SEUs) were first predicted in 1962 [22]. Ironically, they were originally predicted as an unavoidable consequence of the trend in shrinking electronic device sizes, which was predicted to make devices susceptible to SEUs via terrestrial radiation environments. Nevertheless, a continually growing body of research has been dedicated to this particular reliability concern since the latter half of the 1970s. SEUs (in digital devices and circuits) are soft-errors where the state of a device is changed by the transient current produced via the mechanism previously discussed. They are less well-defined in analog circuits, and more difficult to quantify, given the continuous nature of analog signals [23]. Beyond SEU, there exist single-event failure mechanisms which are great reliability concerns to high power devices, such as GaN HEMTs. These failure mechanisms can result in the complete destruction of a device, potentially catastrophic system failure, and can include single-event latchup (SEL), single-event gate rupture (SEGR), single-event burnout (SEB), and others. 


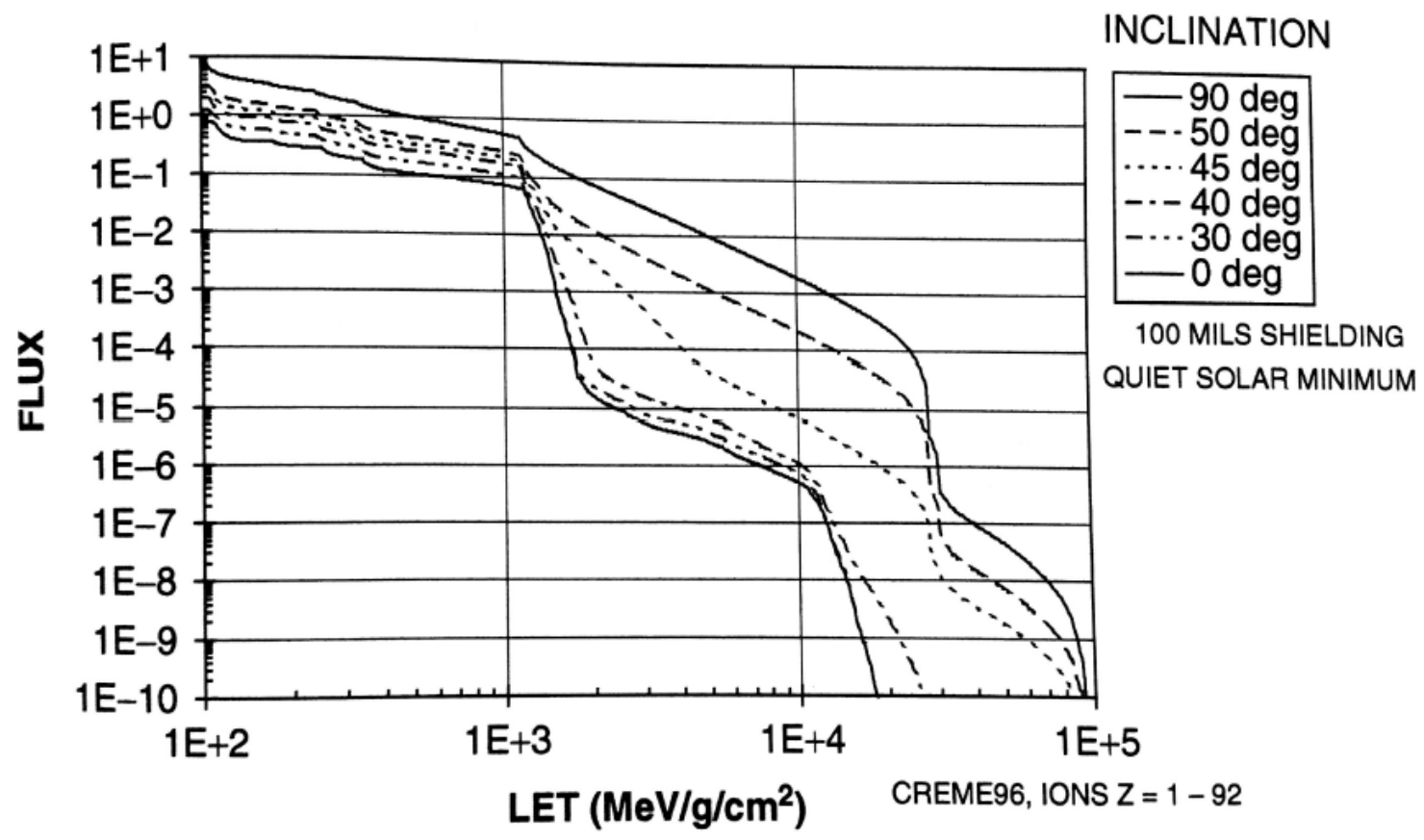

Figure II.6: LET Spectra of heavy ions at LEO $(500 \mathrm{~km})$ at various inclinations. Note that shielding is most significant at the equator (0 degrees inclination). After [19].

\section{II.3.1 Single-Event Charge Collection}

One of the most fundamental metrics for quantifying various aspects of single-event phenomena is an energetic particle's linear energy transfer (LET). LET is defined as the average amount of energy an energetic particle loses per unit path length through a material:

$$
L E T=-\frac{1}{\rho} \frac{d E}{d x}
$$

The dimensionality of this quantity is typically equal to $\mathrm{MeV} \cdot \mathrm{cm}^{2} / \mathrm{mg}$. The $1 / \rho$ term and the unusual units allow LET to be reported as independent of the target material. Obviously, LET will vary with distance as the particle slows and eventually stops in a sufficiently long target, but device sensitive volumes are often small enough that the portion of the path length the ion will travel through the volume is small compared to its range. Under this assumption, LET is generally approximated as constant. Note that so far, this equation is only useful in predicting the average energy deposited in a given device. To calculate the amount of charge generated by this energy deposition, a material parameter must be known that relates the amount of deposited energy it takes 

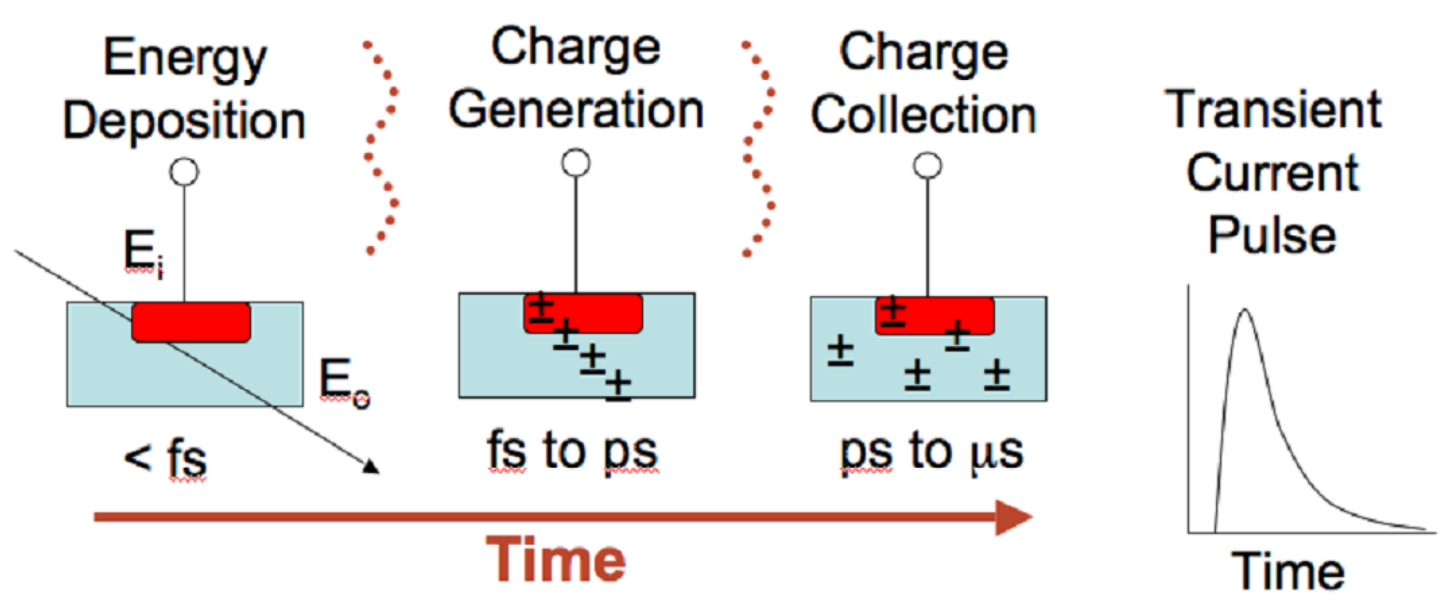

Figure II.7: Simple illustration of the charge generation process. Left to right across the figure: an incident ion deposits energy; the energy liberates charge (electron-hole pairs); some charges move to the device terminal; collected charge appears as transient current. After [25].

to generate one electron-hole pair. The equation to determine generated charge, $Q_{G e n}$ then becomes:

$$
Q_{G e n}=\frac{\rho(L E T) l q}{G}
$$

Where $G$ is the amount of energy required to generate an electron-hole pair in the material (3.6 eV for silicon, $8.9 \mathrm{eV}$ for $\mathrm{GaN}$ [24]), $l$ is the path length, in $\mathrm{cm}$ when using the conventional units of LET, and $q$ is the elementary charge. For silicon, this equation shows that an incident ion of LET $=100 \mathrm{MeV} \cdot \mathrm{cm}^{2} / \mathrm{mg}$, generates approximately $1 \mathrm{pC} / \mu \mathrm{m}$, a relatively convenient number to commit to memory.

Depending on the individual circumstances (device, geometry, operating conditions, etc.), this generated charge can be collected by the device terminals and appear as a transient current, as illustrated in Fig. II.7. This is the process that culminates in a device-level SEE, which can range from a disruptive, yet relatively benign, single-event upset (SEU), to total device destruction and/or system failure.

\section{II.3.2 Single-Event Gate Rupture}

Single-event gate rupture (SEGR) is a potent example of the potential damage SEEs can cause. A SEGR is the single-event induced catastrophic failure of the gate dielectric [26]. Since it is a mechanism that is irreconcilably destructive, unlike effects such as latchup where power can 
be cycled to avoid damage, relatively few experimental investigations have been made into the mechanisms of SEGR. Nonetheless, device simulations have linked hole pileup at the gate oxide to a transient increase in electric field [26]. Fig. II.8 illustrates hole pileup at a gate oxide along an ion strike.

The holes only remain crowded at the oxide interface momentarily, but this collection of positive charges across a dielectric from the negatively-biased gate causes a transient increase in oxide electric field. It was shown that sufficiently energetic ions could push the electric field in a device past it's breakdown value, even when the device is operated within limits set by the manufacturer [27].

\section{II.3.3 Transient Response Measurement}

Given that single-event effects are necessarily initiated by the generation and collection of charge in the device, study of the underlying mechanisms, often though measurements of radiation-induced single-event transients, has proven to be fertile ground for useful insight into various single-event effects, as well as methods of mitigation. Undertaking this study, however, produces its own unique set of challenges. Transient current pulses such as those initiated by ion strikes are exceedingly short. Pulse durations (in terms of full-width at half-max, or FWHM) in the range of hundreds of picoseconds are common, and can be in the range of tens of picoseconds [1].

Historically, charge collection measurements were made by directly measuring the total timeintegrated charge from an ion strike, and constructing a pulse-height spectrum using a multi-channel analyzer [29]. This type of measurement typically takes advantage of a charge-sensitive preamplifier for integrating the transient current signal. An experiment such as this has its strength in its ease of execution and the relative low cost of the necessary equipment, but such simplicity comes with a price. The information content is limited, since the system can only report one single value representing total collected charge for every transient signal. The pulse-height spectrum may yield some more information, but in general, the information one can glean from such a spectrum is limited to "typical" and "worst-case" responses [1]. Further, common preamplifiers utilized for these experiments have very large series resistance. In the case of a very common example: the Ortec 142 has a series resistance of $100 \mathrm{M} \Omega$. This large series resistance leads to noisy results in the case of "leaky" devices. 


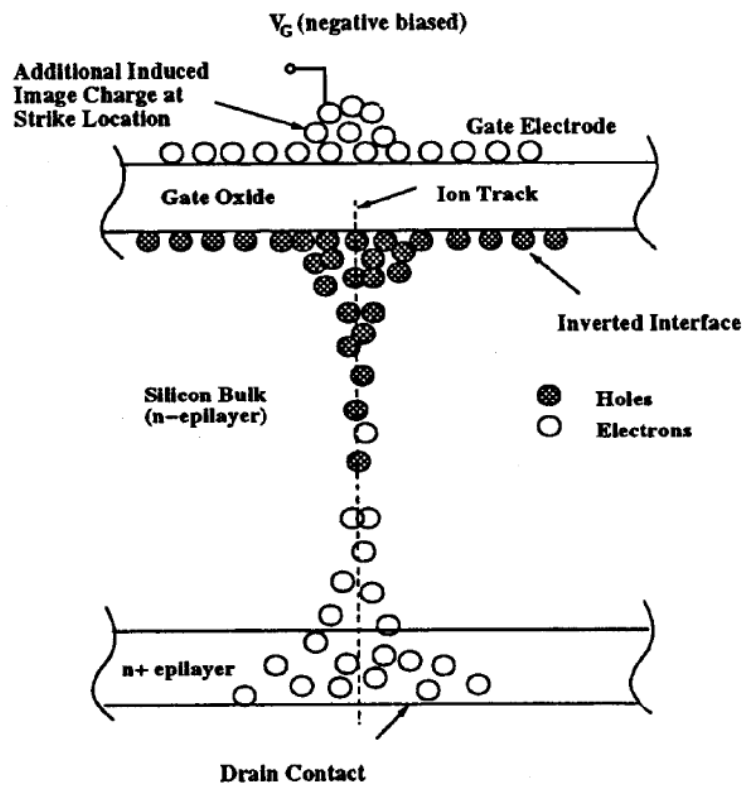

Figure II.8: Simple illustration of the pileup of holes near gate dielectric along the path of an ion strike. Holes are swept up toward the oxide, where the momentarily collect before diffusing laterally to the source. After [28].

By measuring the full transient response, so-called "time-resolved charge collection," an experimenter gains access to a great deal more information. Further, unlike in the case of time-integrated measurements, there is no system calibration required, since the experimenter is making a direct measurement, instead of a constructing a differential spectrum. At face value, time-resolved charge collection is the simpler methodology. The issue lies in the technology available. Until relatively recently, the only way to emulate the high bandwidth necessary to perform time-resolved charge collection measurements was to utilize a sampling oscilloscope, which reconstructs a representative pulse by taking a weighted average of many measurements. The fundamental problem with this methodology is the assumption that the real transient pulses are repeatable. While this is generally a reliable assumption when simulating ion strikes with laser pulses, it is not very reliable when utilizing actual energetic ions, since the device response will depend heavily on strike location. Only relatively recently have single-shot oscilloscopes with sufficient bandwidth and sensitivity become available [1].

Sufficient scope bandwidth, however is still not enough to ensure the integrity of pulse signals on the timescales of ion-induced transient responses. Normal prototyping packages such as dual in-line 


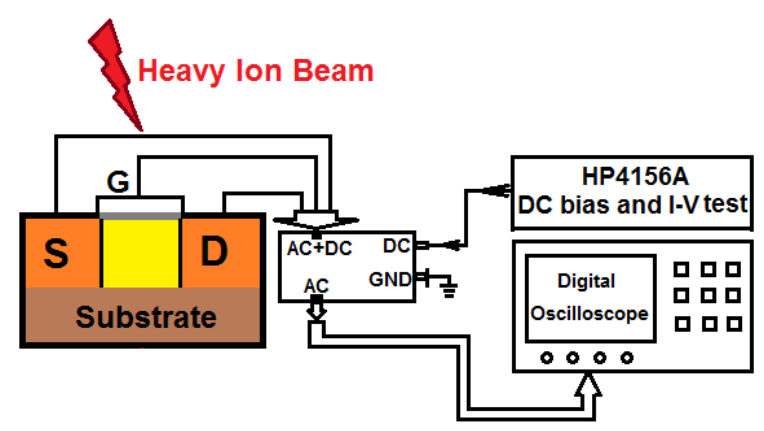

Figure II.9: Block diagram of an example time-resolved charge collection measurement system. Looking from the DUT, the bias tee decouples the AC transient signal from the DC bias.

packages (DIPs) are simply not designed to handle signals in this high-frequency regime. Custom, high-bandwidth packages are often used because they not only provide an excellent ground plane, but they allow the direct attachment of impedance-matched microstrip transmission lines as well as the die itself. These packages are discussed more quantitatively in Section III.2. The system also requires a component to separate the DC bias from the high-speed AC transient signal. Since this component also carries the high-speed signal, it must have highly-controlled bandwidth and be impedance matched. A simplistic block-diagram of a time-resolved testing setup is show in Fig. II.9. The only component this diagram excludes, is a computer to save data from the scope.

In order to illustrate the information that is lost by performing time-integrated charge collection measurements instead of time-resolved, Fig. II.10 shows the same ion-induced current transients plotted on three different timescales. The plot displays a prompt charge collection process dominating at short timescales on the scale of hundreds of picoseconds (top figure), followed by the much longer-lived bi-exponentially decaying charge collection process on timescales greater than nanoseconds (bottom figure).

\section{II.4 Previous Work}

\section{II.4.1 SEEs in Other Material systems}

\section{Gallium Arsenide}

Gallium Arsenide is one of the most common alternative semiconductors, and one of the earliest to be investigated. As such, single-event research in GaAs devices dates back to the early 1980s [30]. In the early charge collection work on GaAs, simple device structures were used, that 

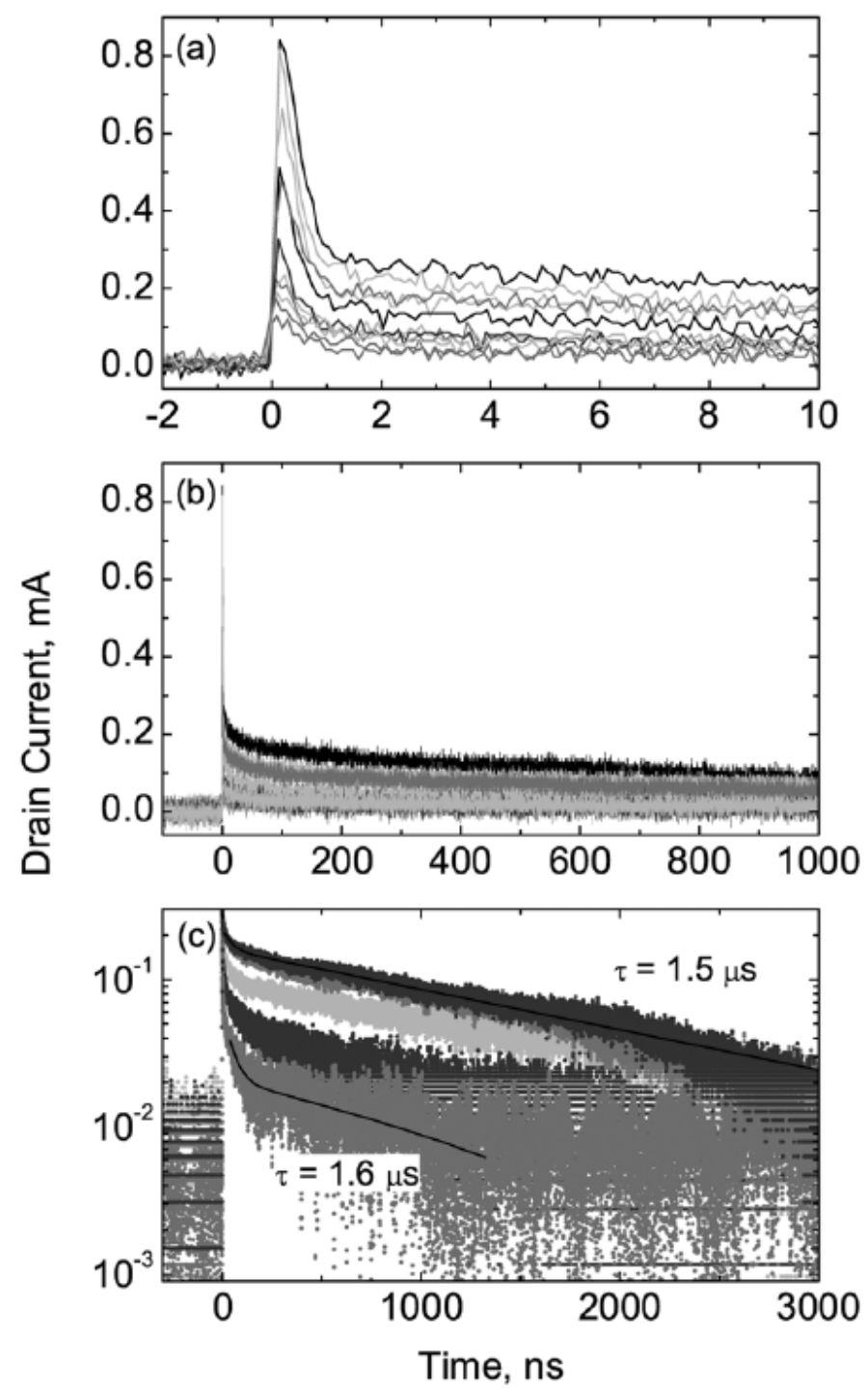

Figure II.10: Ion-induced current transients in InAlAs/InGaAs HEMTs, plotted on three different timescales. Note the change in dominant contributing mechanism at different times. After [1].

were not necessarily any different from their silicon counterparts, as they included only p-n junction and Schottky-barrier diodes [31] (as opposed to later work, which investigated more complex heterostructure-based devices). This early work reported interesting results regarding the so-called "field funneling" effect. This phenomenon, the name for which some dismiss as inappropriate or misleading, occurs when an incident ion of normal incidence to a reverse-biased junction collapses the depletion region of the junction and "stretches" the electric field along the particle track thereby increasing the total charge collection volume [32]. The field funneling effect is visualized in Fig. 


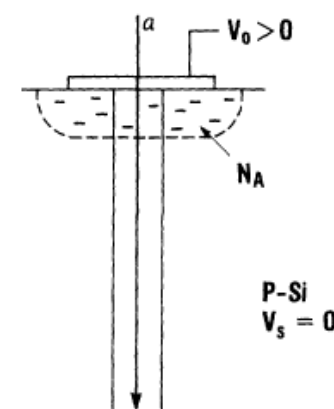

(a)

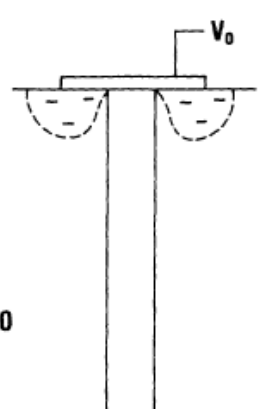

(b)

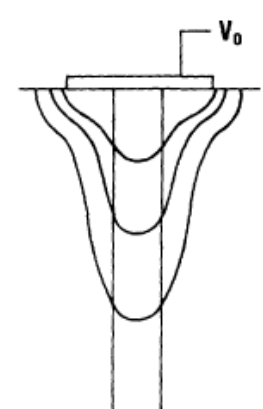

(c)

Figure II.11: Illustration of the so-called "field funneling effect." (a) An alpha particle strikes a pn-junction, then (b) the depletion region collapses, and (c) the potential is modulated along the particle track. After [32].

II.11.

It was noted in an early investigation on various GaAs diode structures, that in the case of devices fabricated on semi-insulating (SI) GaAs substrates, the "field funneling" effect did not contribute significantly to the total collected charge, as is the case in silicon and GaAs devices fabricated on semiconducting (SC) GaAs substrates [31]. Semi-insulating substrates refer to substrates that have a very high resistivity, on the order of a perfectly undoped substrate, which are more easily achievable in practice due to the stoichiometric nature of GaAs [33]. SI substrates provide the advantage that devices separated by only substrate material are relatively well-isolated. The absence of contribution from "field funneling" to the collected charge in SI GaAs devices is advantageous, not only because this leads to less overall collected charge, but because predicting SEU susceptibility is made simpler by excluding "field funneling" from the calculation. The absence of a "field funneling" contribution in SI GaAs devices is illustrated in Fig. II.12.

Another important aspect of GaAs technology that has been subject to numerous investigations is charge collection enhancement. Charge collection enhancement is a phenomenon observed when the measured collected charge is higher than the calculated maximum amount of charge that an incident ion can generate in a sensitive volume. "Field funneling" is one example of such a phenomenon, but other mechanisms have been investigated and identified in GaAs devices. Energetic ions, almost invariably the radiation source used to study charge collection enhancement, can severely modulate voltage potentials in a device, leading to enhancement mechanisms such as source/substrate, and source/channel barrier lowering, where incident radiation sufficiently modu- 


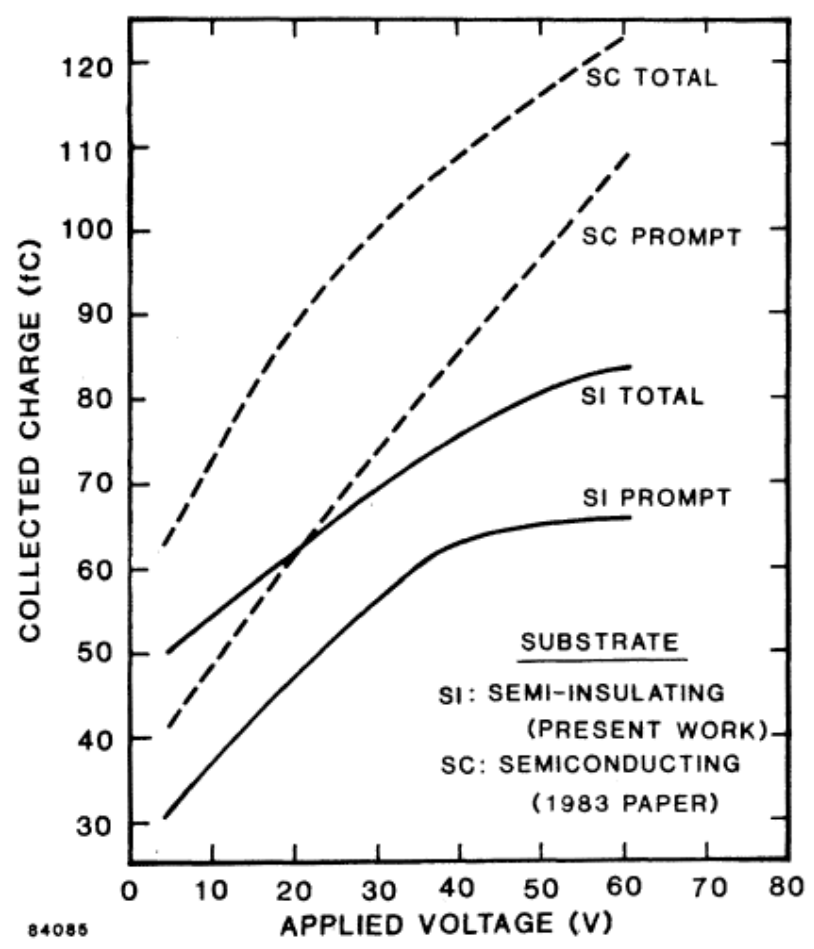

Figure II.12: Collected charge measurements in semiconducting and semi-insulating GaAs. The higher collected charge in SC GaAs is attributed to the greater contribution of "field funneling" in the semiconducting substrate. After [31].

lates these junctions such that current can be injected or collected across them [34].

Charge enhancement mechanisms in GaAs technology led to unexpected results regarding their SEU hardness. Since it had been established before the first SEU measurements were made on GaAs devices, the rad-hardness of GaAs was predicted to extend to a hardness against SEU. Early work demonstrated that this was not the case, as the error-rates predicted by the measurements were comparable to bulk Si technology [35]. In this study, this error-rate was determined to be a consequence of unexpectedly-low upset LET thresholds, i.e., the minimum LET an incident ion must possess to induce an upset. The most probable cause for this low LET threshold is the presence of charge collection enhancement mechanisms.

\section{Other III-V Material Systems}

In addition to the devices described so far, devices with increasingly complex material systems are continually being designed and investigated for a wide variety of specific applications. Complex material systems allow designers to finely tune devices for precisely defined operating character- 


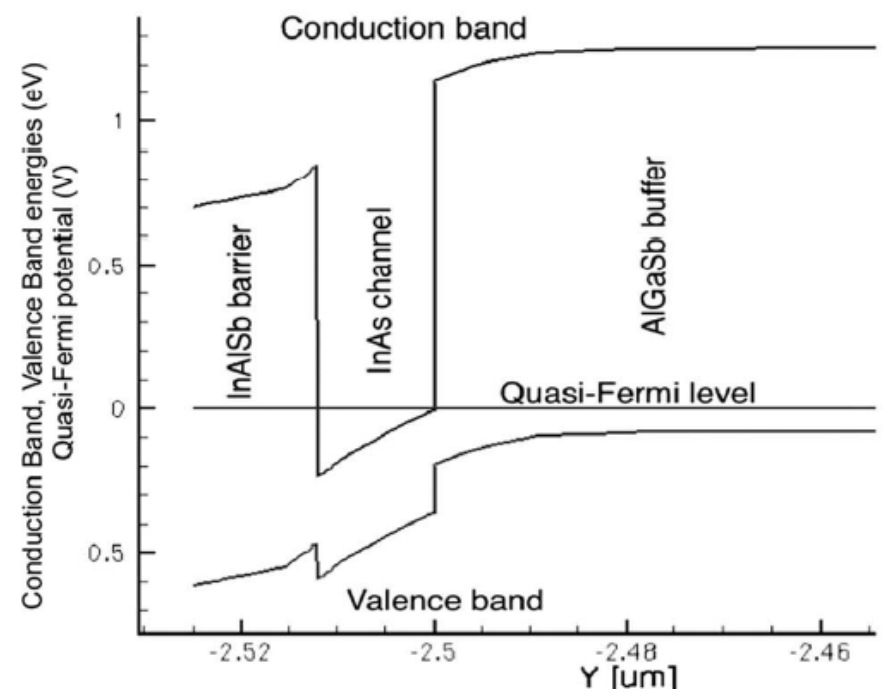

Figure II.13: Vertical cross-section equilibrium band diagram of InAlSb/InAs/AlGaSb HEMT. After [36].

istics or niche applications, and include several completely different materials, or carefully varied stoichiometric combinations of the same material. Contrasting the two-material system described by the AlGAN/GaN HEMTs described above, for example, antimonide-based devices can involve a variety of different materials, as well as exceedingly complex alloys.

The band diagram for an InAlSb/InAs/AlGaSb HEMT is shown in Fig. II.13. This band diagram illustrates the relatively small bandgap of the InAs channel, as well as the large band offsets formed by the heterojunction. Two charge collection mechanisms present in this device are particularly interesting: charge collection under the drain pad, and charge collection enhancement [36]. After previous tests had noted anomalously large single-event charge collection cross-section, the spatial dependence of strike location was measured. The new results and device simulations showed that the depletion region between the $\mathrm{AlGaSb}$ buffer and the $\mathrm{Pd} / \mathrm{Pt} / \mathrm{Au} / \mathrm{Al} / \mathrm{Ga} / \mathrm{Sb} / \mathrm{In} / \mathrm{As}$ alloy drain was capable of collecting a significant amount of charge. This particular results draws attention to the fact that the alloyed materials necessary to fabricate even the source and drain of devices with exotic materials can have an unexpected effect on their single-event response.

The mechanism behind the charge collection enhancement in these devices was determined to have a nature similar to those in the GaAs FET devices as described above (source/channel barrier lowering), however, due to the more complex nature of the device, this effect is highly dependent upon the bias condition of the device, and is delicately balanced such that it is only evident when 


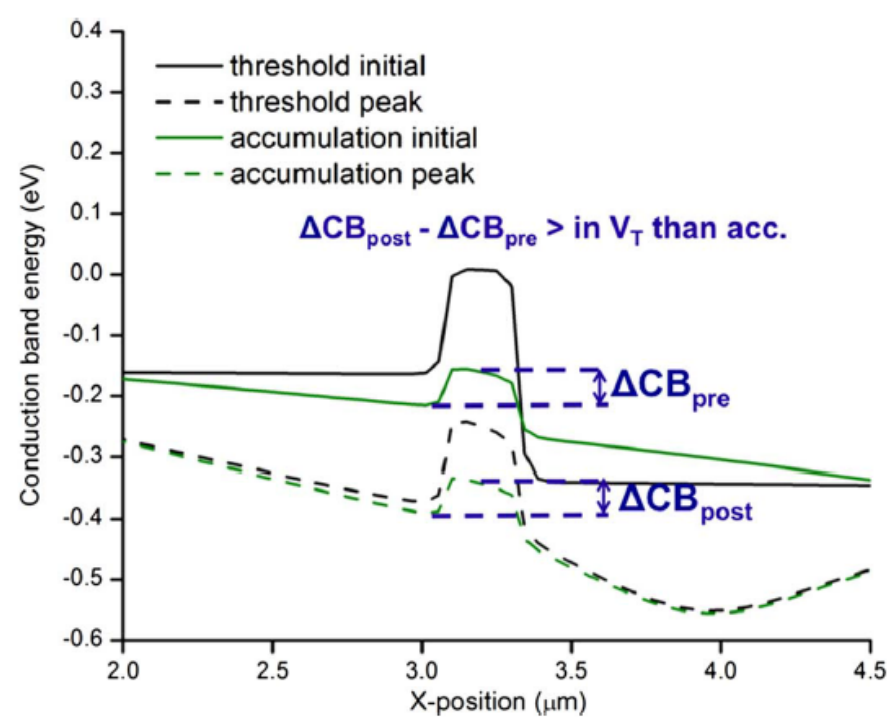

Figure II.14: "Barrier lowering pre- and post strike in threshold and accumulation, obtained from a horizontal cutline through the center of the channel" [36].

the gate voltage is near threshold. This delicate balance is illustrated in Fig. II.14, and is further supported by the very narrow bandgap of the channel material, as shown in Fig. II.13.

This delicate gate bias dependence illustrates the potential difficulty in developing SEU mitigation strategies for devices with this material, and for other materialistically complex devices. Further, the work of [36] provides a good example of the crucial nature of detailed numerical device simulation to understanding the single-event response of non-silicon materials. The level of detail in device simulation is also of critical importance to work of this nature, as the duration of transient pulses in these devices requires careful attention to sources of parasitic impedances from measurement setups.

\section{Silicon Carbide}

Silicon carbide is another composite semiconductor that is well suited for high power and high voltage applications, with some devices operating up to $10 \mathrm{kV}$ [37]. Like its wide-bandgap counterparts, it has demonstrated an extreme resistance to radiation damage [38]. Though SEE information on $\mathrm{SiC}$ devices is sparse, the experiments that have been carried out show interesting results with similarities to other composite semiconductors. The work in [39] studies the charge collection mechanisms in $4 \mathrm{H} \mathrm{SiC} \mathrm{MESFETs} \mathrm{by} \mathrm{irradiating} \mathrm{them} \mathrm{with} \mathrm{heavy} \mathrm{ions} \mathrm{of} \mathrm{various} \mathrm{LETs.} \mathrm{Transient}$ signals produced by ion strikes when the device is in the ON-state are shown in Fig. II.15. 


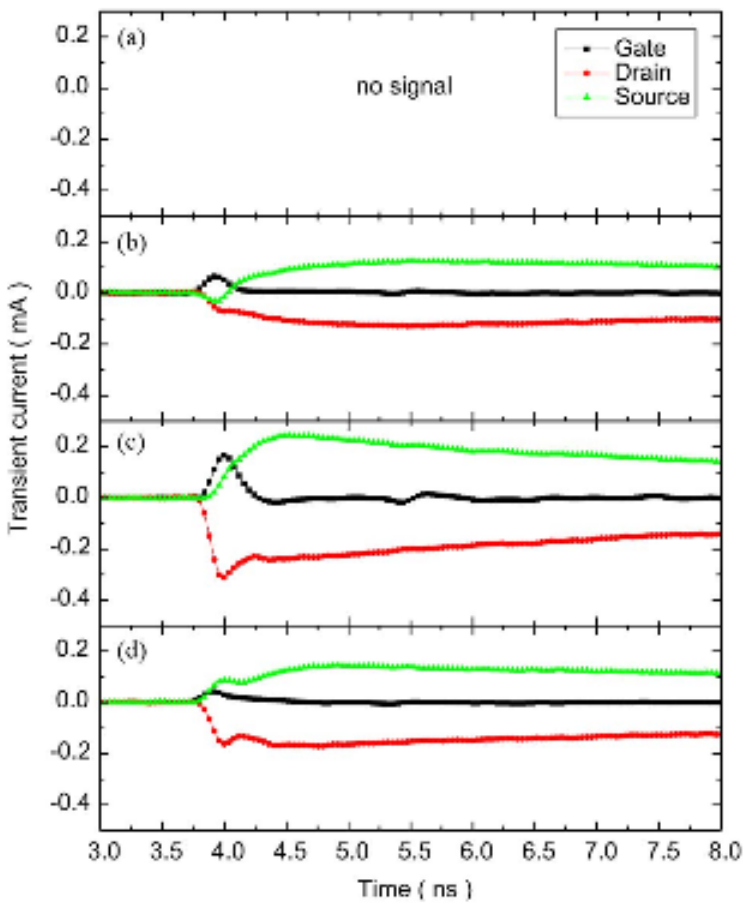

Figure II.15: Current transients observed during ion strikes in SiC MESFETs, with device biased in ON-state. Strike location is (a) in between gate and source, (b) at the gate, (c) in between gate and drain, (d) at the drain. After [39].

Fig. II.15 illustrates an important aspect of the charge collection mechanisms at work, specifically enhancement mechanisms: in each strike that produces a signal, the source and drain current can be seen to "turn on" after the strike, and the resulting transient lasts orders of magnitude longer than the current transient in the gate, which is very short because it is not enhanced. This mechanism is quite similar to the channel modulation mechanisms reported in GaAs devices studied in [34]. However, it is important to note that the charge enhancement factors (the ratio of charge generated by the ion strike to the total collected charge) reported for the $\mathrm{SiC}$ devices was much higher than those reported for GaAs devices ( $\sim 2000$ compared to $\sim 20)$. This is a consequence of the highpower and high-voltage nature of the device, which was biased at voltages an order of magnitude higher than the GaAs MESFETs they are being compared to.

Destructive SEEs are of great concern in $\mathrm{SiC}$ devices, because of their very high operating voltage. High voltage diodes such as SiC Schottky diodes can be particularly susceptible to SEB, which is related to avalanche breakdown; an ionizing particle can generate enough carriers in the high electric field region of a reverse biased diode to essentially short the device terminals via avalanche 

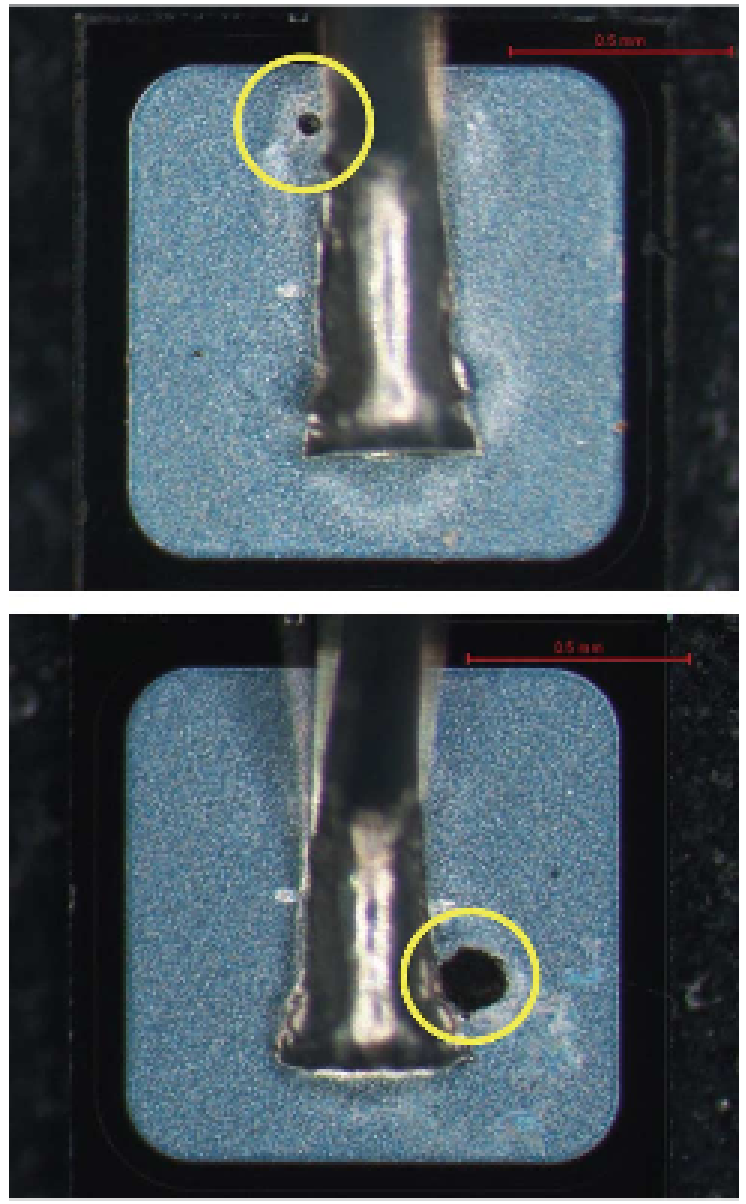

Figure II.16: The bond pads on this $\mathrm{SiC}$ Schottky barrier diode, and the burnout traces circled, show the material destruction caused by neutron-induced SEB. After [40].

multiplication. The work in [40] deals with neutron-induced SEB. In neutron-induced SEB, the incident neutron liberates secondary ionizing particles that initiate avalanche breakdown. The consequences of this breakdown are illustrated in Fig. II.16, which shows the destructive nature of this failure mechanism. Complicating the effort to quantify and understand this phenomenon, diodes of comparable operating parameters but different parts manufacturers exhibited SEB susceptibility that were different by orders of magnitude. Monte Carlo simulations pointed to the conclusion that the highly energetic carbon atoms liberated by incident neutrons play a key part in the SEB mechanisms at work. 


\section{II.4.2 AlGaN/GaN HEMTs}

In the substantial collection of literature on single-event effects in microelectronics devices and circuits, very few exist on GaN-based devices such as GaN HEMTs. Further, the studies that are available at this time are not mechanism-focused, but rather are instead aimed in the direction of parts qualification. No studies could be found on SEEs in GaN MOS-HEMTs.

Since one of the advantageous applications of GaN HEMT technology is to high-voltage and high-power devices, it is instructive to compare the SEE reliability of GaN HEMTs to similarlyapplied silicon technologies. The work referenced in [10] uses a military-standard (MIL-STD750D) bench test for evaluating the susceptibility of a commercial GaN HEMT, and compares its performance to high-voltage silicon MOSFETs (commercially-produced HEXFETs). Specifically, the researchers were investigating single-event burnout (SEB) and single-event gate rupture (SEGR), though it was noted in the work that the lack of an insulating oxide layer in the GaN HEMTs will prvent the device from failing by the same mechanisms as the MOSFETs.

The devices in [10] were irradiated with neutrons and heavy ions of LET, in silicon, ranging from $1.8-60 \mathrm{MeV} \cdot \mathrm{cm}^{2} / \mathrm{mg}\left(1.3-47 \mathrm{MeV} \cdot \mathrm{cm}^{2} / \mathrm{mg}\right.$ in $\left.\mathrm{GaN}\right)$. The experimental setup was verified by recording failure statistics of the silicon MOSFETs and constructing corresponding cross-sections curves as a function of LET. Failures were observed in silicon MOSFETs at LET as low as $18 \mathrm{MeV}$. $\mathrm{cm}^{2} / \mathrm{mg}$ (523 MeV Fe ions). Failure in MOSFETs was also observed during $14 \mathrm{MeV}$ neutron radiation.

Consonant with gallium nitride's previously demonstrated robustness, the GaN devices tested in [10] showed far fewer failures than the silicon devices tested. No failures or functional changes were observed during neutron, $139 \mathrm{MeV} \mathrm{N}$ ion $\left(\mathrm{LET}=1.3 \mathrm{MeV} \cdot \mathrm{cm}^{2} / \mathrm{mg}\right.$ in GaN), nor $523 \mathrm{MeV}$ Fe ion $\left(\mathrm{LET}=14 \mathrm{MeV} \cdot \mathrm{cm}^{2} / \mathrm{mg}\right.$ in $\left.\mathrm{GaN}\right)$ irradiation. Further, while the authors noted both SEB and SEGR failure mechanisms in the silicon MOSFETs, none of the failures in the GaN devices exhibited this failure mode. Instead, the authors believed the few failures exhibited by the GaN devices displayed behavior analogous to SEGR, as shown in Fig. II.17, which shows damage modes under $236 \mathrm{MeV} \mathrm{Br}$ ion $\left(\mathrm{LET}=32 \mathrm{MeV} \cdot \mathrm{cm}^{2} / \mathrm{mg}\right.$ in $\left.\mathrm{GaN}\right)$ irradiation. The stair-step appearance of the figure illustrates the dramatic increases in gate current induced by interaction with a single energetic particle. 


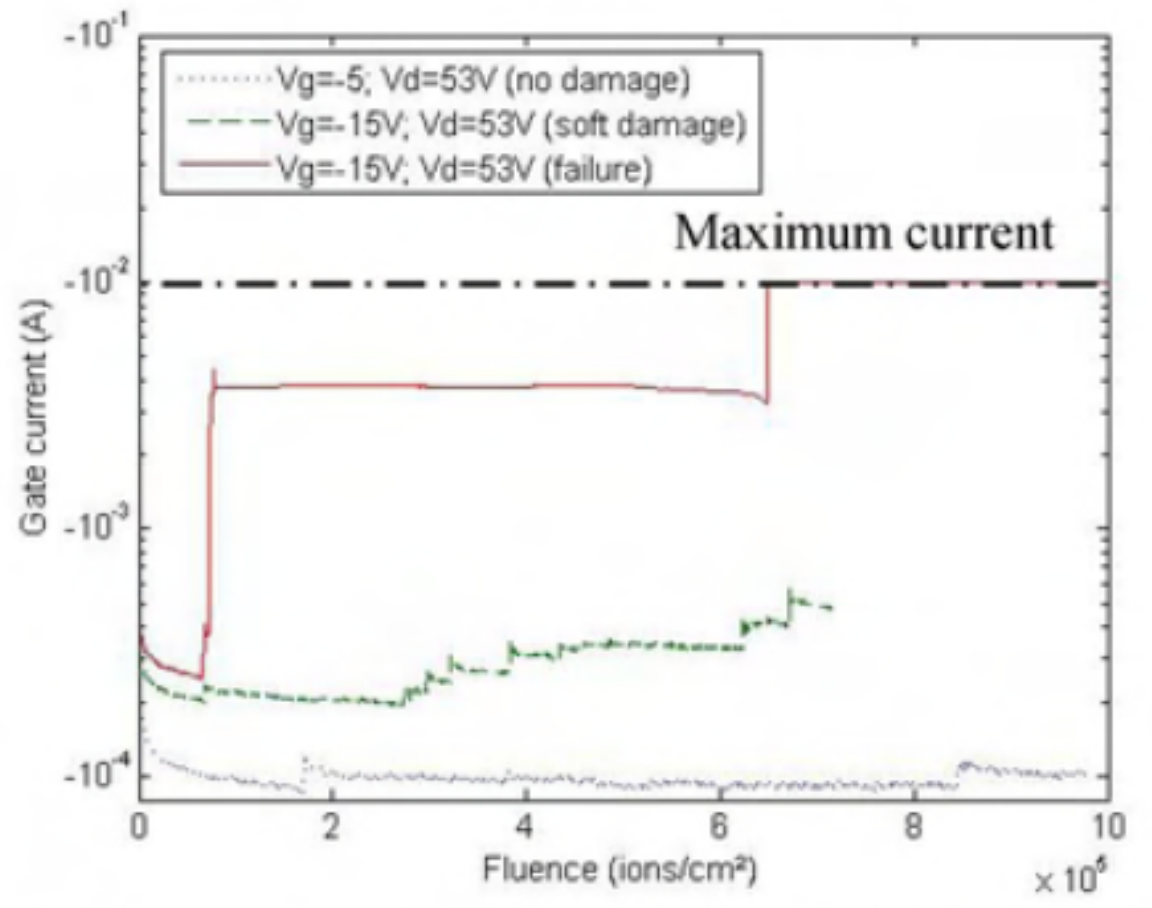

Figure II.17: Gate current in GaN HEMTs under $236 \mathrm{MeV}$ Br irradiation. Note that device under lower $-5 \mathrm{~V}$ gate bias demonstrated little change in gate current, while the $-15 \mathrm{~V}$ bias condition produced both "soft" degradation in gate current, as well as total failure. After [10]. 


\section{CHAPTER III}

\section{Experimental Methodology}

\section{III.1 Device Description}

Each device under test (DUT) was an AlGaN/GaN HEMT or MOS-HEMT grown on a highlyresistive silicon substrate. Fig. III.1a shows a schematic cross-section of the device, which is described in further detail in [6]. The gate electrode is 300/2000/500 $\AA \mathrm{Ni} / \mathrm{Au} / \mathrm{Ni}$. A layer of $25 \%$ $\mathrm{Al} \mathrm{AlGaN}$ is followed by a $1 \mu \mathrm{m}$ layer of (unintentionally doped) GaN. A $1 \mu \mathrm{m}$ layer of GaN serves as a transition to the resistive silicon substrate. The MOS-HEMT devices have a $4 \mathrm{~nm}$ insulating layer of either $\mathrm{HfO}_{2}$ or $\mathrm{Al}_{2} \mathrm{O}_{3}$ deposited between the $\mathrm{AlGaN}$ layer and gate electrode. The twodimensional electron gas (2DEG) is formed in the GaN buffer layer, adjacent to the AlGaN, shown by the red and black dashed line in Fig. III.1a. Fig. III.1b shows a typical band diagram of an $\mathrm{AlGaN} / \mathrm{GaN}$ HEMT near the 2DEG at zero-bias. The band diagram is along a one-dimensional cutline through the center of Fig. III.1a that runs vertically under the gate and through the AlGaN and $\mathrm{GaN}$.

The structures of interest are arrays of two HEMT or MOS-HEMT devices, with two source contacts and shared drain and gate contacts. The drawn gate lengths are either $3 \mu \mathrm{m}$ (MOS devices) or $4 \mu \mathrm{m}$ (Schottky device). Each device has a source-to-gate and drain-to-gate spacing of $2 \mu \mathrm{m}$. DC characteristics for an $\mathrm{HfO}_{2}$-gate device are shown in Fig. III.2. The layout of a single structure (two HEMT devices) is shown in Fig. III.3. Note that each device is normally on unless a negative gate bias is applied, and in general $\left|V_{T, \mathrm{Al}_{2} \mathrm{O}_{3}}\right|>\left|V_{T, \mathrm{HfO}_{2}}\right|>\mid V_{T}$, Schottky $\mid$. Since the devices are developmental in nature, variation is seen in threshold voltages, even with devices of the same gate/gate-oxide type. $\mathrm{V}_{G E}$ is taken as the effective gate voltage and $\mathrm{V}_{G E}=\mathrm{V}_{G}-\mathrm{V}_{T}$.

\section{III.2 Pulse Capture Setup}

The test setup employed for this study is constructed to measure a high-speed transient signal with a real-time oscilloscope. The total collected charge is determined by numerically integrating the signal waveform captured by the oscilloscope over time. Similar setups have been used in similar work $[1,36,41,42]$ to capture radiation-induced transient response of devices. 


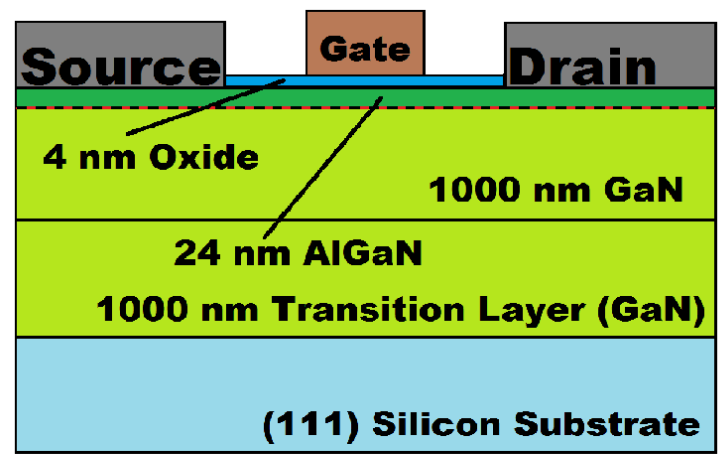

(a)

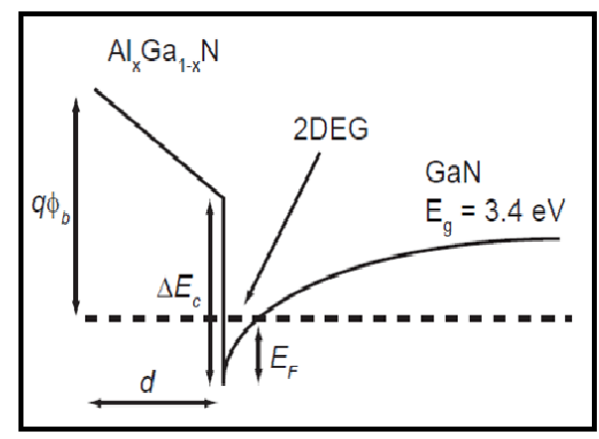

(b)

Figure III.1: (a) Schematic cross-section of AlGaN/GaN MOS-HEMT. (b) Typical band diagram of $\mathrm{AlGaN} / \mathrm{GaN}$ interface showing 2DEG.

For all tests, the device under test was mounted on a custom-milled metal package fabricated with microstrip transmission lines and $2.92 \mathrm{~mm} \mathrm{~K}$-connectors. A similar custom package is shown in Fig. III.4. The microstrip transmission lines are thin gold conductors separated from the ground plane by a dielectric substrate. They are attached via conductive epoxy directly to the metal package. For a given connection to the device, short bondwires run from the bondpads on the die to the microstrip conductor. Conductive epoxy connects the microstrip to the package-mounted Kconnector, which consists of a stress-relieving connection and a flange launcher, both of which are impedance-matched to the rest of the system and have a bandwidth of $40 \mathrm{GHz}$.

The microstrip transmission lines are impedance-matched to the rest of the system (50 $\Omega$ characteristic impedance), and are characterized by $<1 \mathrm{~dB}$ loss at frequencies up to $30 \mathrm{GHz}$. To reduce the effects of parasitic inductance between the bondwires coming off the chip and the microstrip transmission lines, the bondwire lengths were kept as short as reasonably possible. Similar packaging schemes have been characterized more quantitatively in [43]. All cables and connections in the 


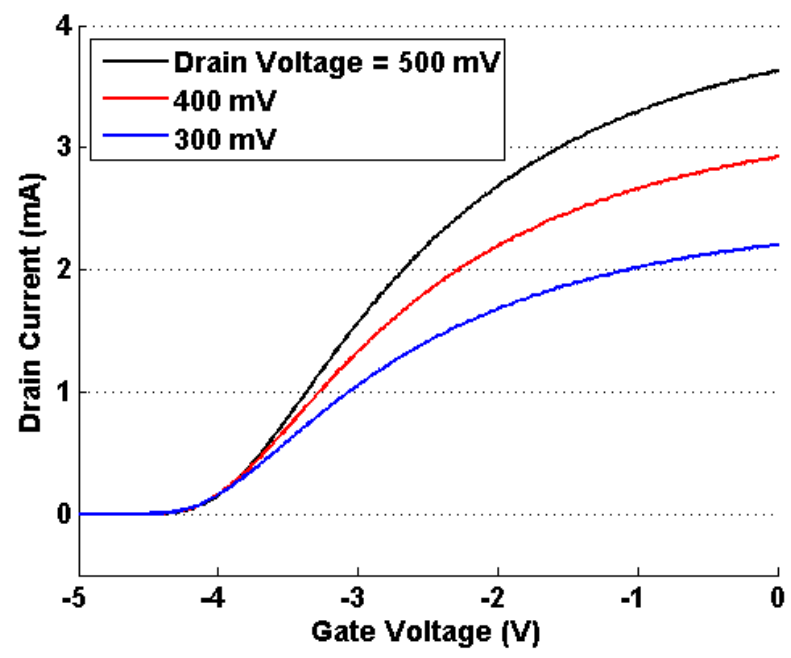

Figure III.2: DC characteristics of $\mathrm{HfO}_{2}$-gate DUT. Drain Current is measured as a function of gate voltage. Current measurements are shown for three drain voltage conditions.

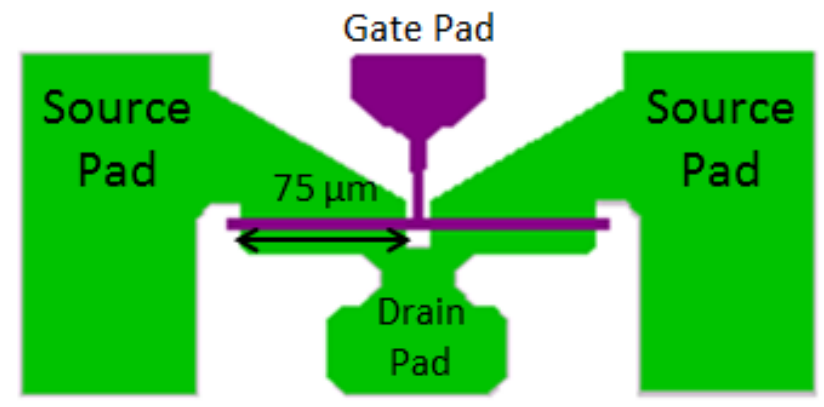

Figure III.3: Layout mask of AlGaN/GaN DUT. Two devices share a gate and drain, while having separate source contacts.

signal path are rated for bandwidths exceeding $24 \mathrm{GHz}$. The signal path components were chosen to have a matched $50 \Omega$ impedance from the microstrip transmission lines to the front end of the oscilloscope used for the measurements. As discussed in the Background section, the accurate characterization of every component in the signal path is crucial for making an accurate measurement.

S-parameters can be used to infer information about parasitics, attenuation, and reflection. Fig. III.5, shows the S-parameters of a custom-milled high speed package composed of identical signalpath components to those used in the experimental work, including gold bondwires, microstrip transmission lines, and K-connectors. The frequency response of the high speed packages was evaluated with an Agilent PNA-X N5245A programmable network analyzer. Two terminals were 


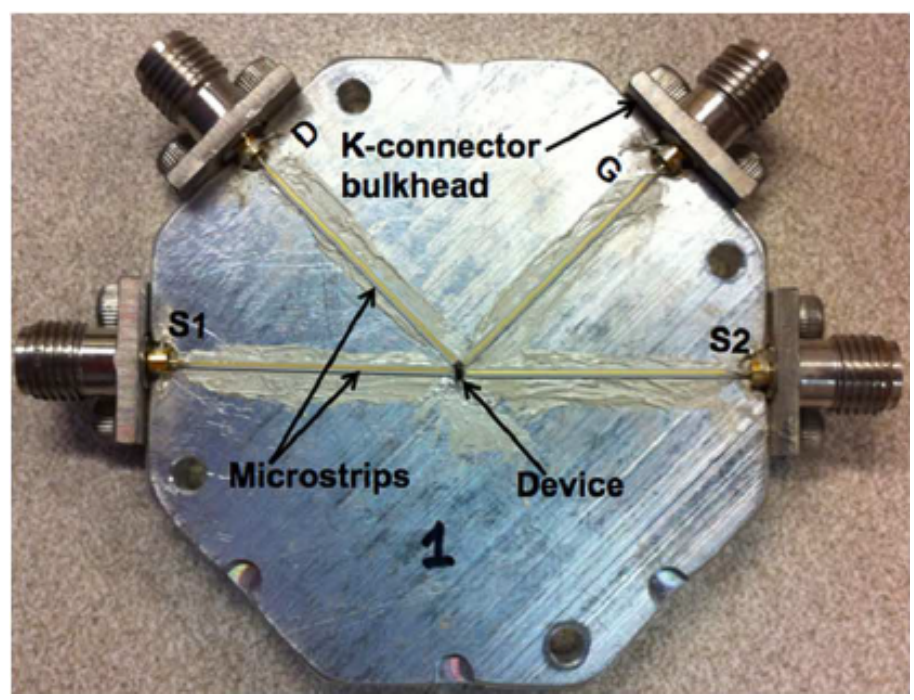

Figure III.4: Custom-milled high-speed package fabricated at Vanderbilt University specifically for time-resolved charge collection measurements. Note the microwave transmission lines carrying the signal to the bulkheads. After [36].

connected through a bondwire with similar length to that of the DUT connected between Port A (input) and Port B (output). In the figure, $S_{11}$ and $S_{22}$ represent the input and output port reflection coefficients, respectively; $S_{21}$ and $S_{12}$ represent the forward and reverse transmission coefficients, respectively. At frequencies up to $25 \mathrm{GHz}$, the insertion loss $\left(-\mathrm{S}_{21}\right)$ is $<3 \mathrm{~dB}$, and the reflection coefficients at each port $\left(\mathrm{S}_{11}\right.$ and $\left.\mathrm{S}_{22}\right)$ are $<-10 \mathrm{~dB}$. The quality of the signal path at high frequencies is further supported by the time-domain reflectometry (TDR) measurement shown in the insert [44].

The DC bias for each terminal of the device is provided through a Picosecond Pulse Labs model 5542-219 bias-tee (50 $\Omega$ characteristic impedance, $40 \mathrm{GHz}$ minimum bandwidth, 7 ps risetime). The bias tee decouples the high-speed transient signal (AC) and the power supply (DC) to avoid unnecessary stress on the scope. An Agilent 4156 parameter analyzer is used periodically to monitor the health of the device and to record any shift in threshold voltage. In each experiment, DC bias is applied to the gate and drain, and the source is connected to ground. The backside of the die is attached via conductive epoxy to the metal package; the metal package is grounded for all measurements.

The charge collection measurements performed are time-resolved, meaning they are calculated based on recording the full transient current pulse induced by an ion. The recorded transients are then processed using in-house software written specifically for handling data of this type: R.E.R. 


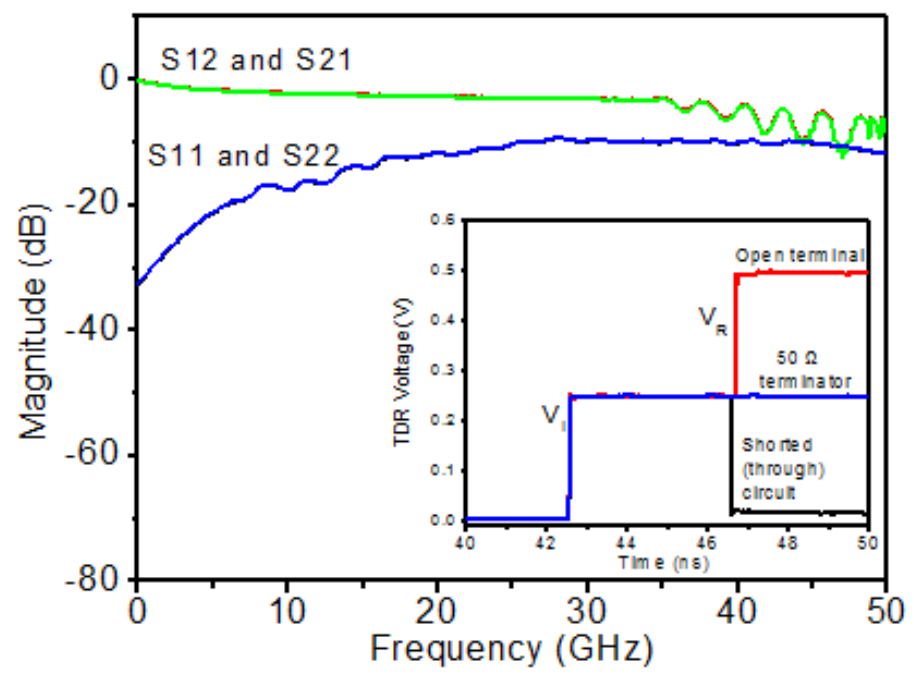

Figure III.5: S-parameters of custom-made high-speed package as a function of frequency up to 50 GHz. $S_{11}$ tracks $S_{22}$ almost exactly, and $S_{12}$ tracks $S_{21}$ almost exactly. The insert figure is the TDR response of the same package. After [44].

Automated Transient Capture and analysis (RATACA). RATACA allows the user to apply parameterized filters to eliminate noise from the captured signals, however, due to the proximity of the succeeding measurements to the noise level, this function was not used in this work. RATACA also allows "selective integration" of the transient current pulse, which handles one transient pulse at a time to pick out the peak (positive or negative), and determine reasonable values for integration bounds around the signal (for example, the nearest points at which the signal has a value of zero). The selective integration feature greatly reduces the noise in these charge collection measurements. Current pulses are integrated in time to yield the collected charge value. 


\section{CHAPTER IV}

\section{Charge Collection Measurements}

\section{IV.1 Broadbeam Irradiation}

\section{IV.1.1 Experimental Setup}

Transient events were induced by strikes of $14.3 \mathrm{MeV}$ oxygen ions provided by Vanderbilt University's Pelletron electrostatic particle accelerator, supplying an average flux of $5 \times 10^{8}$ particles/s$\mathrm{cm}^{2}$. The beam spot diameter is approximately $3.8 \mathrm{~cm}$, significantly larger than the size of the device. Beam spot size was measured via $\mathrm{X}$ and $\mathrm{Y}$ raster-scans at the end of the beamline with a $0.03 \mathrm{~cm}^{2}$ aperture surface-barrier detector in $5 \mathrm{~mm}$ steps. Results were compared with backscattering measurements to determine relative intensity independent of beam current. All measurements were made at room temperature. $14.3 \mathrm{MeV}$ oxygen has an LET of $5.4 \mathrm{MeV} \cdot \mathrm{cm}^{2} / \mathrm{mg}(\mathrm{GaN})$ and a projected range of $9.7 \mu \mathrm{m}$ in $\mathrm{GaN}$ [45]. Calculations from the Monte Carlo Radiative Energy Deposition (MRED) code [45] confirm that the thin metal layers and passivation on these devices have minimal effects on the incident ion energy.

Transients were measured in real-time using a Tektronix TDS6124C oscilloscope with a frontend bandwidth of $12 \mathrm{GHz}$. Under the operating conditions described (measuring all four channels), the sampling rate is $20 \mathrm{GS} / \mathrm{s}$, resulting in a measurement resolution of $50 \mathrm{ps}$ per data point. DC bias was provided by an Agilent 4156A parameter analyzer.

The sampling rate of the scope and speed of the transients limit the information that can be inferred directly from the shape of the transient signal. The two scopes used in this work have maximum resolution of 50 and 20 ps per data point, meaning a 100 ps transient pulse will have, at most, five data points. This inherently limits how well the scope will reproduce the transient signal. However, this work qualitatively investigates the mechanisms of charge collection in these devices, as opposed to quantifying the device response. Though the electrical characteristics of the measurement setup (parasitic capacitance and inductance, reflection, scope limitations, etc.) will have some impact on the shape and magnitude of the transient current signal, these factors do not significantly affect the conclusions of this study. 


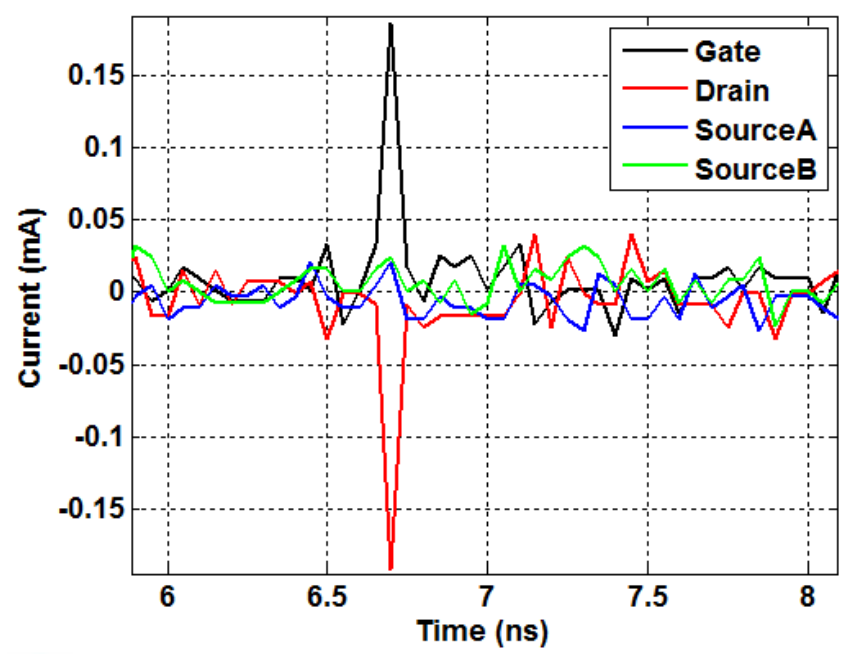

Figure IV.1: Drain and gate current transients produced in the DUT by $14.3 \mathrm{MeV}$ oxygen ions: $\mathrm{V}_{G E}=-0.6 \mathrm{~V}, \mathrm{~V}_{D}=500 \mathrm{mV}, \mathrm{V}_{S A}=0 \mathrm{~V}, \mathrm{~V}_{S B}=0 \mathrm{~V}$. Note the symmetry of the gate and drain signals, and the absence of any transient signal on the source channels.

\section{IV.1.2 Results}

In the $\mathrm{HfO}_{2}$-gate DUT, current transients were observed exclusively at the gate and at the drain.

Fig. IV.1 shows a typical current transient on each of the four oscilloscope channels. The bias configuration in Fig. IV.1 is a hard-off state. The threshold voltage $\mathrm{V}_{T}$ of this depletion-mode device is $-4 \mathrm{~V}$. In all cases the drain and gate currents were approximately equal and opposite to within the resolution of the measurements. The voltage signal from the scope was converted to current using the $50 \Omega$ characteristic impedance of the scope input. Despite the high beam flux, the number of detectable transient events was extremely low. In this work, a detectable transient event refers to a recorded trigger on an oscilloscope, always on the channel connected to the gate, as discussed in more detail below. Seconds to minutes passed between oscilloscope triggers. After only a small number of transients are recorded, device performance begins to degrade, likely due to displacement damage. Consequently, very few transient events could be recorded at each bias point $(\sim 10)$, far too few to report statistically significant information regarding bias dependence.

Charge collection efficiency observed was on the order of $10 \%$. This approximate charge collection efficiency was calculated using the integrated transient current signal from the scope, taking into account the charge deposited by the ion into the AlGaN and GaN layers, as calculated using MRED [45]. Charge collection enhancement is not observed in the broadbeam data (or for the 
microbeam data).

The triggering channel was originally set to be the drain, in anticipation of transient current between the source and drain. The channel was switched to the gate to better examine the observed gate transient current [46]. Triggering on the gate later proved crucial in subsequent tests of Schottky gate devices, as discussed in section III-D. Given the size of the noise compared to the signal being measured, the trigger was set as low as possible, just above the $2.5 \mathrm{mV}$ noise floor of the testing environment. No transient current was observed between the source and drain.

\section{IV.2 Microbeam Irradiation}

\section{IV.2.1 Experimental Setup}

Devices from the same lot were tested using the Heavy Ion Microbeam at Sandia National Laboratories [47] using a $48 \mathrm{MeV}$ silicon ion beam, with LET of $10 \mathrm{MeV} \cdot \mathrm{cm}^{2} / \mathrm{mg}(\mathrm{GaN})$ [45], and an elliptical spot-size where full-width at half-max $(\mathrm{FWHM})(\mathrm{x})=1 \mu \mathrm{m}$ and $\operatorname{FWHM}(\mathrm{y})=2.4 \mu \mathrm{m}$. The small, magnetically-focused spot-size of the beam allows collection of positional information over the entire device structure with resolution equal to the respective (x) and (y) FWHM. After exiting the Van de Graaff tandem accelerator, the beam is scanned in the $\mathrm{x}$ and $\mathrm{y}$ directions over the surface of the device electrostatically [47]. In the case of the Schottky gate device, the substrate is monitored by the scope via conductive epoxy on the backside of the die to capture any substrate transients. The backsides of the MOS devices were grounded to the package as they were in the broadbeam experimental setup.

Transients were measured in real-time using a Tektronix DPO72004 oscilloscope with a frontend bandwidth of $16 \mathrm{GHz}$. Under the operating conditions described (measuring all four channels), the sampling rate is $50 \mathrm{GS} / \mathrm{s}$, resulting in a measurement resolution of $20 \mathrm{ps}$ per data point. The devices were biased using Keithley 2400 power supplies and were periodically monitored by an Agilent 4156C parameter analyzer to verify the functionality of the device.

\section{IV.2.2 Results}

Multiple devices and device structures were investigated using the microbeam. $\mathrm{HfO}_{2}$ gate-oxide devices, Schottky gate devices, and $\mathrm{Al}_{2} \mathrm{O}_{3}$ gate-oxide devices were measured. As observed in the broadbeam experiments, the number of detectable events in the devices with insulating gate oxides 


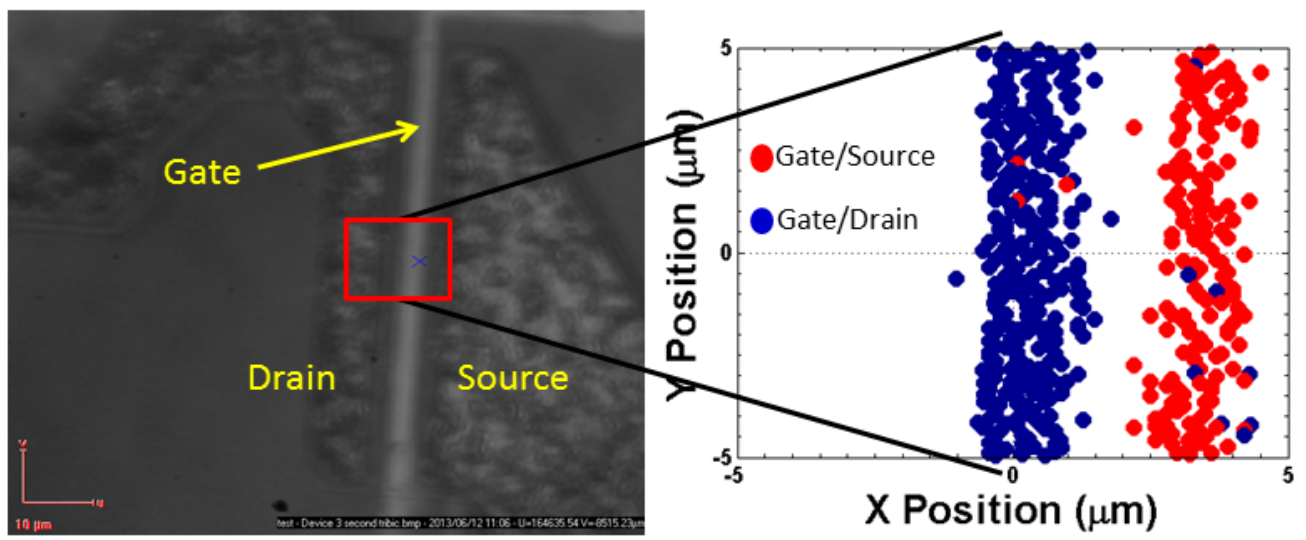

(a)

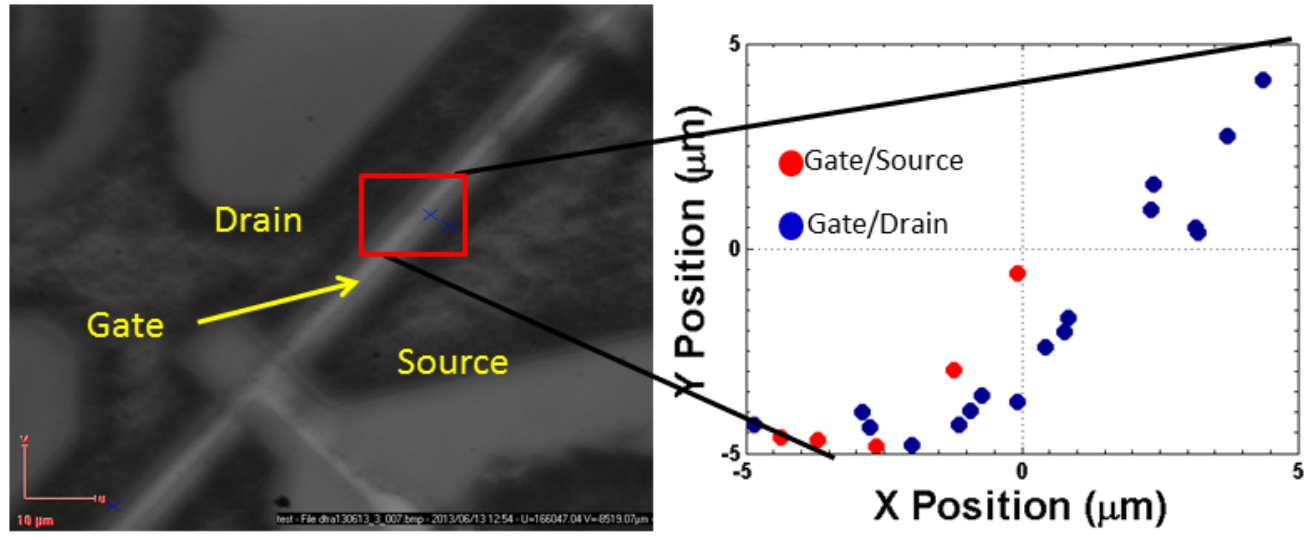

(b)

Figure IV.2: (a) $10 \mu \mathrm{m} \times 10 \mu \mathrm{m}$ area scan of a Schottky-gate device. (b) Comparable area scan of an $\mathrm{HfO}_{2}$-gate device. Each point on each plot represents a detected transient event (gate-channel scope trigger). Note the strong positional dependence on strike location. The number of observed transient events is almost two orders of magnitude greater in the Schottky-gate device compared to the $\mathrm{HfO}_{2}$ gate oxide device.

was very low. In the Schottky devices, the transient pulse appears between the gate and either the drain or source, depending on strike location. No measurable current transients were recorded on the substrate channel. As illustrated in Fig. IV.2a, where every point on the plot represents the location of an oscilloscope trigger on the gate channel, a current transient is most likely to be observed when the ion strike is near either edge of the gate. A few positional outliers can be observed in Fig. IV.2a, likely caused by stray ions from the beam or another rare event. Triggering on the gate proved to be the most reliable channel for each type of device; in no instance was a transient recorded between only the source and drain. As in the broadbeam tests, the charge collection efficiency was on the order of $10 \%$. 


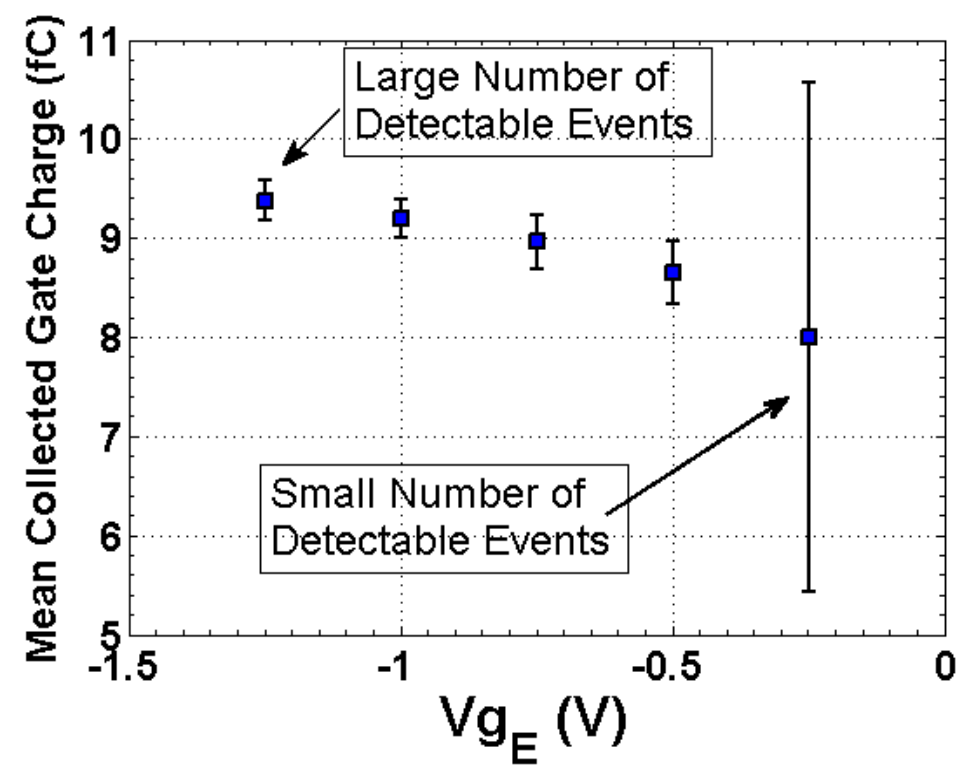

Figure IV.3: Mean collected charge in identical $10 \mu \mathrm{m} \times 10 \mu \mathrm{m}$ area scans of a $4 \mu \mathrm{m}$ gate Schottkygate device. Total collected gate charge trends weakly with increasingly negative gate bias.

A comparison of $100 \mu \mathrm{m}^{2}$ scans with comparable bias conditions and beam flux is shown in Fig. IV.2. Each of the two scans measures either a Schottky-gate or $\mathrm{HfO}_{2}$-gate device. The window is $10 \mu \mathrm{m} \times 10 \mu \mathrm{m}$, and is centered approximately on the center of the gate. The scan covers the entire gate in the (approximate) $\mathrm{x}$-direction, as well as the access regions on either side. The number of transient events observed in the Schottky-gate device, and thus the cross-section for measurable events, is almost two orders of magnitude greater than that of the $\mathrm{HfO}_{2}$ gate oxide device, even taking into account its slightly smaller size. The average peak current of the transients in Schottkygate devices was slightly larger in general. Far fewer gate-source transients were observed in the $\mathrm{HfO}_{2}$ gate oxide device.

Comparably-sized area scans were also performed on the $\mathrm{Al}_{2} \mathrm{O}_{3}$ gate-oxide devices, but zero events were recorded in these scans, as discussed further below. Irradiation of the $\mathrm{Al}_{2} \mathrm{O}_{3}$-gate device was confirmed by other sensitive structures on the die, and device functionality was also confirmed.

In the Schottky-gate devices, both the total number of detected events and the mean collected charge were strongly correlated to gate bias. The relationship of collected charge to gate bias is illustrated in Fig. IV.3. More charge is collected with increasingly negative gate bias. A more complex trend has previously been observed in other types of III-V HEMT devices [36]. The increasing size 
of the error bars at lower voltages, especially the last point at $-0.25 \mathrm{~V}$, underscores that as gate bias approaches the threshold voltage, and the field under the gate decreases, the number of observed transient events falls off sharply. Given that a number of previous studies [36] have concluded maximum charge collection occurs near gate threshold (or pinchoff voltage), some other mechanism is at work. One possible explanation is that the decreasing peak value of the current transient is preventing the scope from triggering. Given the limited temporal resolution of the recorded transients, nothing can be said about the shape of the transients with confidence. If the total collected charge were increasing by pulses spreading out and peak current slightly decreasing, this measurement setup would not be able to resolve it. 


\section{CHAPTER V}

\section{Mechanisms}

\section{V.1 Simulation Setup}

The device was simulated in 2D using Synopsys TCAD device simulation software [48]. The simulated device was verified to have approximately equal DC $\left(\mathrm{I}_{D}-\mathrm{V}_{G}\right)$ characteristics to the real device. The simulated structure is shown in Fig. V.1, with the yellow arrow indicating strike location. The simulated substrate is $20 \mu \mathrm{m}$ of highly resistive silicon. An electrode is placed at the bottom of this substrate and held at ground. The band alignment between $\mathrm{HfO}_{2}$ or $\mathrm{Al}_{2} \mathrm{O}_{3}$ and $\mathrm{AlGaN}$ corresponds to conduction band offsets reported in [7] and [8], respectively. Ion strikes were defined to be Gaussian in time and space (50 $\mathrm{nm}$ track radius, and $1 \mathrm{ps}$ duration). The simulations resulted in transients with comparable temporal profiles to the experimental data. The simulated transients also appeared between the gate and drain or gate and source, consistent with the experimental observations reported above. In each simulation, current transients only appeared at the gate terminal, concurrent with a transient at either the source or the drain, whichever terminal was nearest to the strike.

\section{V.2 Simulation Results}

Fig. V.2 shows the equilibrium band diagram of an $\mathrm{HfO}_{2}$-gate oxide device. The cutline is taken vertically through the device, through the center of the gate, and goes through the oxide, AlGaN, and GaN. The valence band barrier for holes in the channel and AlGaN region to move to the gate is very small $(\sim 0.07 \mathrm{eV})$. Given the increasing amount of charge collected with increasingly negative gate bias, and the small size of the valence band barrier, we conclude that the gate collects holes.

To illustrate the reason for the differences in the number of detectable transient events among the various gate structures, equilibrium band diagrams are presented for the Schottky-gate device in Fig. V.3 and the $\mathrm{Al}_{2} \mathrm{O}_{3}$ gate-oxide device in Fig. V.4. The valence band barrier introduced by the $\mathrm{Al}_{2} \mathrm{O}_{3}$ layer is $\sim 0.8 \mathrm{eV}$, more than an order of magnitude greater than the valence band barrier introduced by the $\mathrm{HfO}_{2}$ layer. Recall that the Schottky-gate devices had the highest number of

detectable events. Simulation results show that these devices also have no valence band barrier 


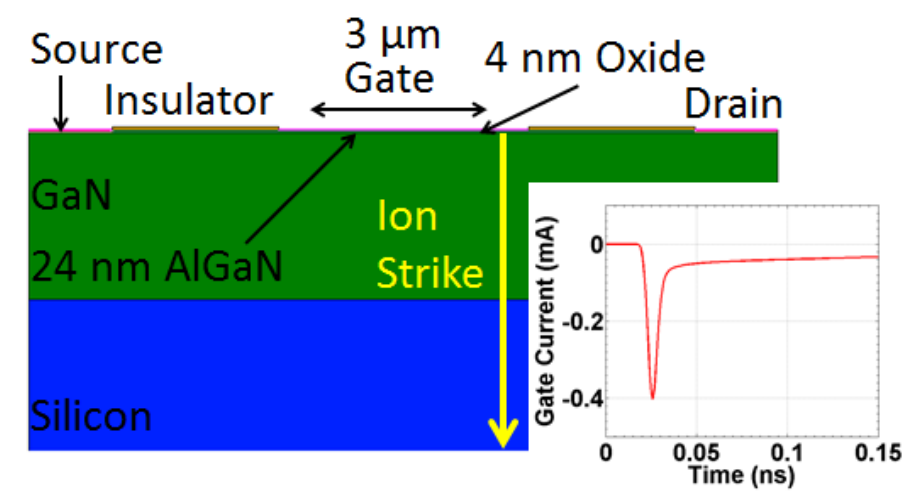

Figure V.1: Simulated device structure showing relevant dimensions and a particular strike location. The resulting gate current transient from such a strike simulation is shown in the inset.

for hole collection at the gate. A comparison of the peak transient current height demonstrates why more events were detected in some devices than others. Fig. V.5 shows typical gate current transients in an $\mathrm{HfO}_{2}$-gate and a Schottky-gate device. On average, the peak current was higher in the Schottky-gate device than the $\mathrm{HfO}_{2}$-gate device. Further, even the largest transients (by pulse height) in the Schottky-gate device are only a few $\mathrm{mV}$ away from the noise floor of the scope (an $8.4 \mathrm{mV}$ signal compared to a $2.5 \mathrm{mV}$ noise maximum). In the $\mathrm{HfO}_{2}$ - and $\mathrm{Al}_{2} \mathrm{O}_{3}$-gate devices, it is likely that there are transient events that are too small to be resolved by the experimental setup.

In the Schottky-gate devices, transient pulses either appeared between the gate and drain, or the gate and source. The mechanism behind this is best illustrated by visualizing the conduction band pre- and post-strike along a horizontal cutline through the channel, along the 2DEG between the source and drain contacts. Fig. V.6 shows this horizontal cutline in a simulated device constructed like the one shown in Fig. V.1, except for the absence of the $4 \mathrm{~nm}$ oxide layer. The red trace in Fig. V.6 shows the conduction band 25 ps after the strike. The strike occurs at the drain-side edge of the gate. It reduces the conduction band energy significantly, but only on the side where the strike occurs, leaving the overall barrier between source and drain intact. The same band reduction occurs near the source when a comparable strike is incident on the source-side edge of the gate. These devices have relatively large $(3 \mu \mathrm{m})$ gates, and the temporal isolation of source and drain transients may not be present in smaller devices.

Beyond the spatial segregation of the gate-drain/gate-source transient responses, there is also spatial separation between the areas where these responses were observed. This fact is evident 


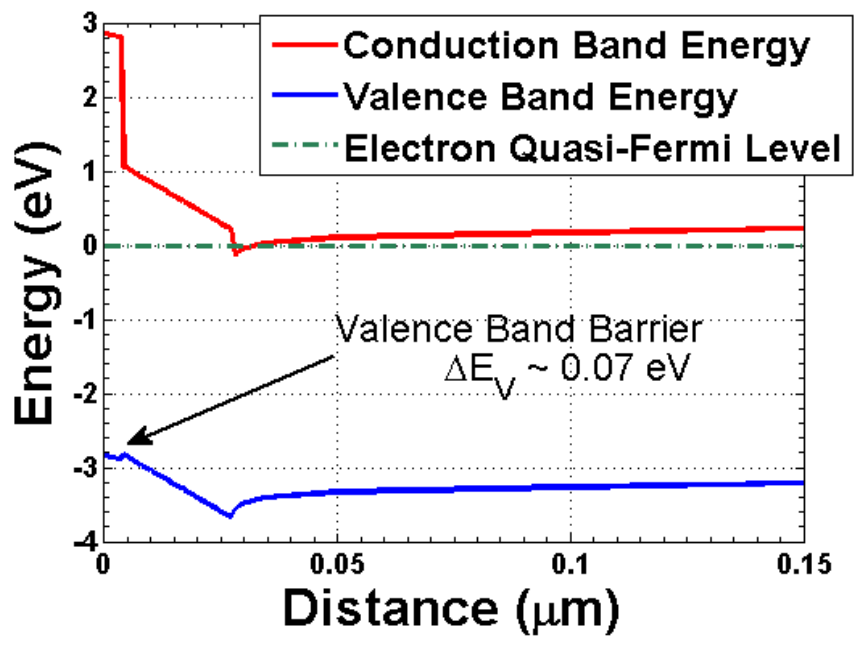

Figure V.2: Equilibrium band diagram of on $\mathrm{HfO}_{2}$-gate device. The oxide layer introduces a slight valence band barrier between the gate and $\mathrm{AlGaN}$ layer.

from Fig. IV.2(b), which shows an empty area between the red and blue band-like regions where ion strikes produced a measurable transient response. The band-like regions spatially correspond approximately to the edges of the gate stack. To address the absence of measurable transient events from strikes in the middle of the gate, the magnitude of the electric field under the gate is shown in Fig. V.7. The vertical cutlines shown are taken at two locations: the center of the gate, and the drain-side edge of the gate (the location of the ion strike in Fig. V.1). The figure focuses in the top of the GaN layer, just under the AlGaN layer. The magnitude of the electric field, graphed horizontally, is peaked near the edges of the gate stack on the drain and source sides, and the field penetrates deeper into the material, as shown in Fig. V.7. A stronger electric field leads to increased electric current, via Ohm's Law, and the transient current that was measured was exceedingly close to the noise level of the system. The presence of an increased electric field deeper into the GaN layer also increases the effective collection depth of an incident ion, relative to the collection depth for a strike in the center of the gate. Consequently, strikes to parts of the device where the electric field is slightly weaker and shallower may not produce transient current of sufficient magnitude to be detectable by the oscilloscope. 


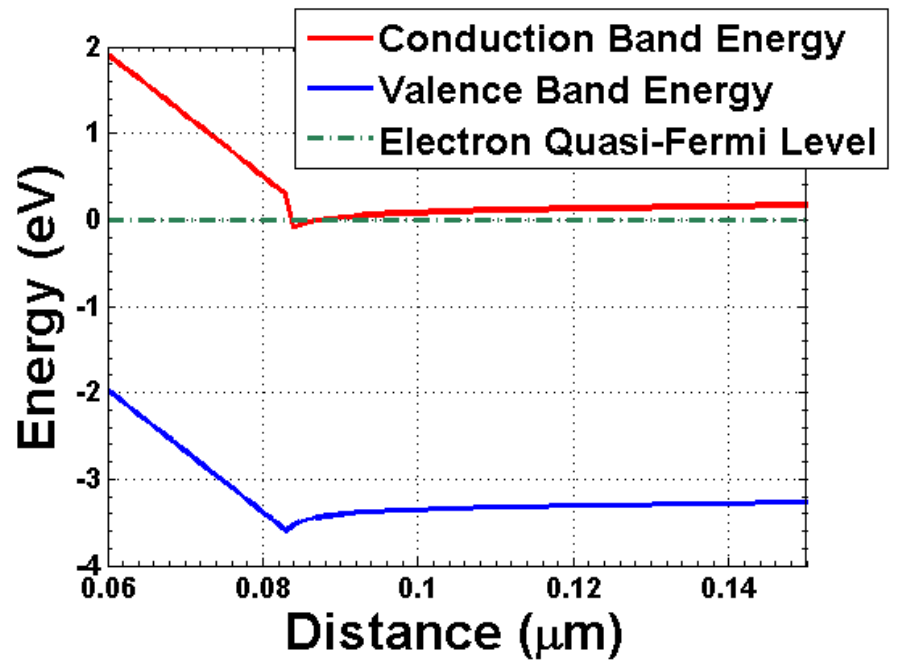

Figure V.3: Equilibrium band diagram of Schottky-gate device. The absence of a gate oxide layer in these devices promotes hole collection at the gate. This is due to the lack of a valence band barrier between the gate metal and the semiconductor material.

\section{V.3 Discussion}

The simulation results show that the band alignment determines how much charge will be collected by the gate. In the simulated Schottky-gate device, the ion strikes result in hole current at the gate terminal. In the case of the $\mathrm{HfO}_{2}$ gate-oxide devices, transient gate current is observed because the valence band barrier is not sufficient to prevent detectable amounts of charge from reaching the gate. In the case of the $\mathrm{Al}_{2} \mathrm{O}_{3}$ gate-oxide devices, this wider-bandgap oxide introduces a more substantial valence band barrier, and the magnitude of the hole current at the gate is much smaller than that simulated in the Schottky or $\mathrm{HfO}_{2}$-gate devices.

The ability of charge carriers to move from the channel to the gate in the event of an ion strike is a phenomenon that could potentially be exploited. The mechanisms of single-event gate rupture (SEGR) are not completely understood [49]; hole pile-up near the interface, however, plays a role by increasing the electric field in the gate oxide. [26]. The absence of hole pile-up in AlGaN/GaN MOS-HEMTs may prove to reduce the SEGR susceptibility. This will be useful to explore in future work. 


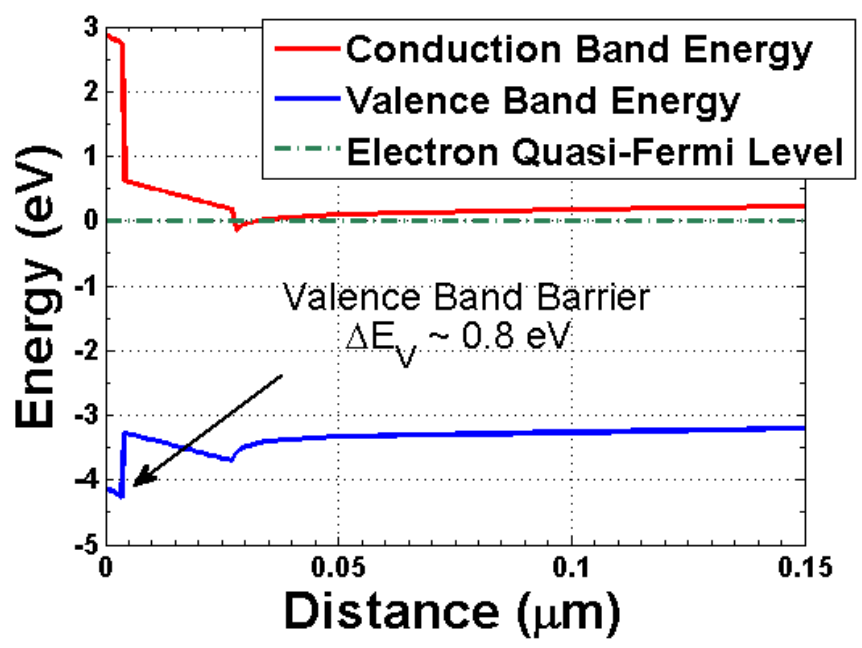

Figure V.4: Equilibrium band diagram of $\mathrm{Al}_{2} \mathrm{O}_{3}$-gate device. The oxide layer introduces significant valence band barrier relative to the barrier in the $\mathrm{HfO}_{2}$-gate device.

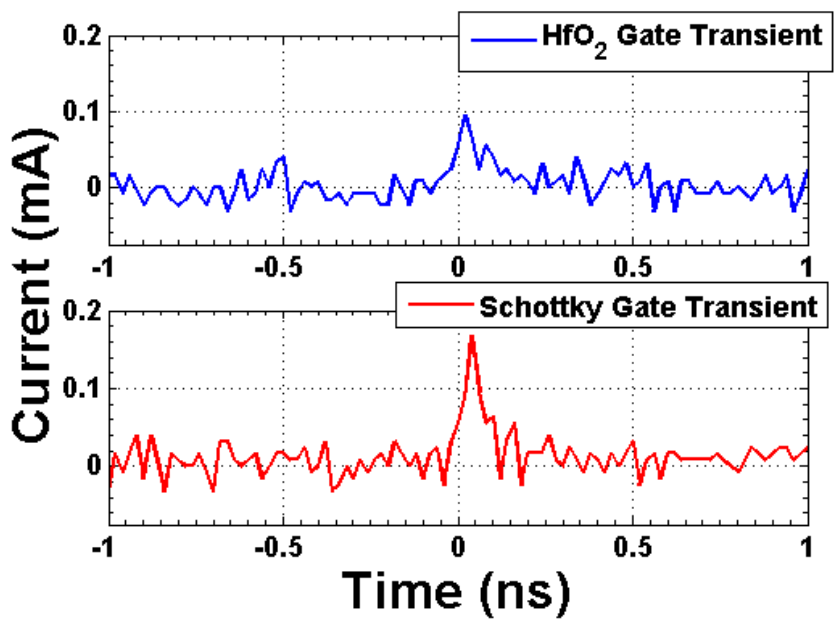

Figure V.5: Direct comparison of typical gate transient currents in $\mathrm{HfO}_{2}$-gate (top) and Schottkygate (bottom) devices. Note the larger peak current in the Schottky-gate device transient. 


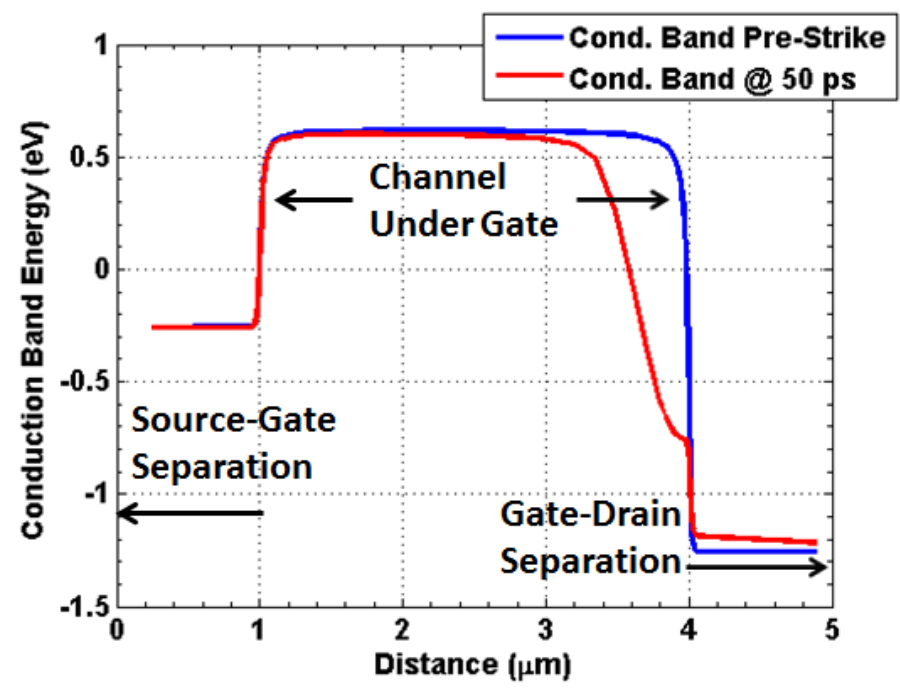

Figure V.6: Conduction band energy along a horizontal cutline following the channel of a Schottkygate device in the source-to-drain direction. The far left and far right regions of the figure are the source-gate and gate-drain separation regions respectively. The strike induces localized conduction band distortion, while the band energy on the side opposite the strike remains largely unchanged. This helps explain the reliable isolation of events in the source or drain as shown in Fig. IV.2.

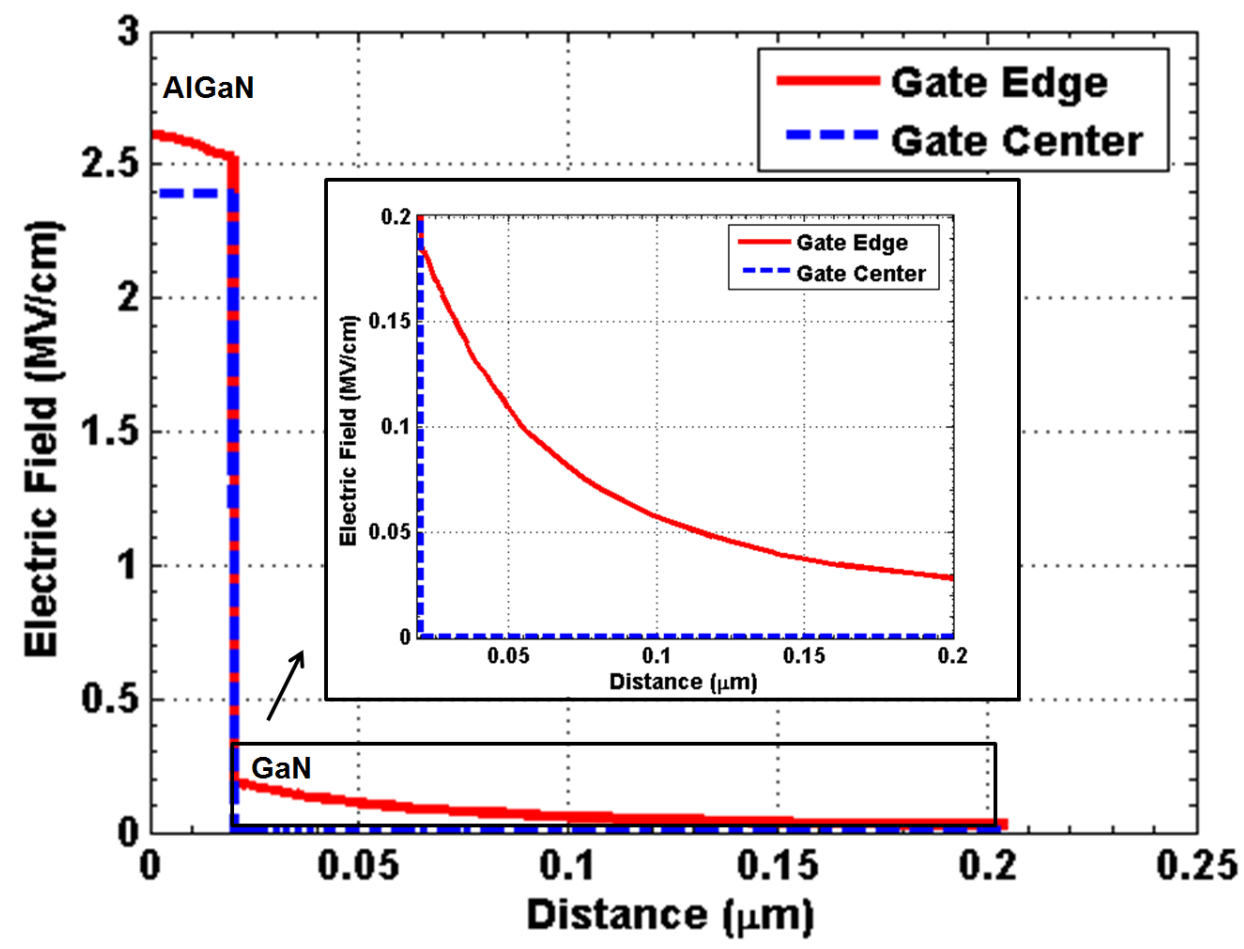

Figure V.7: Magnitude of the electric field along vertical cutlines through the gate of a Schottky-gate device, focused at the top of the GaN layer. The electric field has peaks near the edges of the gate stack, where the increased strength and depth of the field increase the transient current response in those regions. 


\section{CHAPTER VI}

\section{Conclusions}

The goal of this work was to investigate the charge collection mechanisms in AlGaN/GaN MOSHEMTs. This was accomplished experimentally by making time-resolved charge collection measurements with broadbeam and microbeam irradiation. The importance of the gallium nitride material systems, as well as AlGaN/GaN HEMTs was discussed in the background section, with emphasis placed on the advantages and challenges surrounding MOS-HEMTs. The motivation to study charge collection mechanisms was also discussed, from the standpoint of single-event effects. The advantages and challenges of making time-resolved charge collection measurements involving picoseconds-long transients were also presented in the background section.

The AlGaN/GaN MOS-HEMTs (with $\mathrm{HfO}_{2}$-insulated gates) and HEMTs examined exhibited significant charge collection at the gate, concurrent with charge collected at either the drain or source. The physical mechanism behind this behavior was investigated with Synopsis TCAD device simulation software. Device simulations show that the gate charge collection is a result of the band structure of the device, which promotes hole collection at the gate. The presence of the oxide layer decreases the number and peak current of detectable transient events in MOS devices relative to comparable Schottky-gate devices. Concern was expressed regarding the possibility that the experimental setup used in this work may not be sensitive enough to resolve diminished transients from the noise, especially in the case of the MOS-HEMT devices.

Transient current between only the drain and source was not observed. The ion-strike induced potential modulation in the channel is spatially limited to the region near the strike. This fact, coupled with the relative large gate length of the device, leads to transient current appearing at either the gate/drain or gate/source.The spatially limited potential modulation prevents any other current path. Gate oxide material and band alignment play a key role in the charge collection mechanisms

of these MOS-HEMT devices. The ability of the gate oxide to conduct transient hole current may reduce susceptibility to some failure mechanisms common in power FETs. 


\section{BIBLIOGRAPHY}

[1] D. McMorrow, V. Ferlet-Cavrois, P. Paillet, O. Duhamel, J. Baggio, J. B. Boos, and J. S. Melinger, "Transient response of semiconductor electronics to ionizing radiation: Recent developments in charge-collection measurement," IEEE Trans. Nucl. Sci., vol. 54, no. 4, pp. 1010-1017, 2007.

[2] S. M. Khanna, J. Webb, H. Tang, A. J. Houdayer, and C. Carlone, "2 MeV proton radiation damage studies of gallium nitride films through low temperature photoluminescence spectroscopy measurements," IEEE Trans. Nucl. Sci., vol. 47, no. 6, pp. 2322-2328, 2000.

[3] T. Yamasaki, Y. Kittaka, H. Minamide, K. Yamauchi, S. Miwa, S. Goto, M. Nakayama, M. Kono, and N. Yoshida, "A 68\% efficiency, C-band 100W GaN power amplifier for space applications," in 2010 IEEE MTT-S International Microwave Symposium Digest (MTT), 2010, pp. $1384-1387$.

[4] X. Hu, A. P. Karmarkar, B. Jun, D. M. Fleetwood, R. D. Schrimpf, R. D. Geil, R. A. Weller, B. D. White, M. Bataiev, L. J. Brillson, and U. K. Mishra, "Proton-irradiation effects on AlGaN/AlN/GaN high electron mobility transistors," IEEE Trans. Nucl. Sci., vol. 50, no. 6, pp. 1791-1796, 2003.

[5] B. Luo, J. W. Johnson, F. Ren, K. K. Allums, C. R. Abernathy, S. J. Pearton, A. M. Dabiran, A. M. Wowchack, C. J. Polley, P. P. Chow, D. Schoenfeld, and A. G. Baca, "Influence of ${ }^{60} \mathrm{Co}$ gamma-rays on dc performance of AlGaN/GaN high electron mobility transistors," Appl. Phys. Lett., vol. 80, no. 4, pp. 604-606, 2002.

[6] O. I. Saadat and T. Palacios, "Low temperature gate dielectric deposition for recessed AlGaN/GaN MIS-HEMTs," in Proc. of the European Solid-State Device Research Conference (ESSDERC), 2011, pp. 287-290.

[7] C. Liu, E. F. Chor, and L. S. Tan, "Investigations of $\mathrm{HfO}_{2} / \mathrm{AlGaN} / \mathrm{GaN}$ metal-oxidesemiconductor high electron mobility transistors," Appl. Phys. Lett., vol. 88, no. 17, p. 173504, 2006.

[8] T. Hashizume, S. Ootomo, T. Inagaki, and H. Hasegawa, "Surface passivation of GaN and GaN/AlGaN heterostructures by dielectric films and its application to insulated-gate heterostructure transistors," J. Vac. Sci. Technol. B, vol. 21, no. 4, pp. 1828-1838, 2003.

[9] S. Kuboyama, A. Maru, H. Shindou, N. Ikeda, T. Hirao, H. Abe, and T. Tamura, "Singleevent damages caused by heavy ions observed in AlGaN/GaN HEMTs," IEEE Trans. Nucl. Sci., vol. 58, no. 6, pp. 2734-2738, 2011.

[10] S. Bazzoli, S. Girard, V. Ferlet-Cavrois, J. Baggio, P. Paillet, and O. Duhamel, "SEE sensitivity of a COTS GaN transistor and silicon MOSFETs," in Proc. 9th European Conference on Radiation and Its Effects on Components and Systems, 2007, pp. 565-569.

[11] H. Morkoç, in Nitride Semiconductors and Devices. Springer-Verlag Berlin Heidelberg, 1999, pp. 8-39. 
[12] H. Morkoç, A. Di Carlo, and R. Cingolani, "GaN-based modulation doped FETs," in LowDimensional Nitride Semiconductors, B. Gil, Ed. Oxford University Press, 2002, pp. 341414.

[13] U. K. Mishra, P. Parikh, and Y.-F. Wu, "AlGaN/GaN HEMTs-an overview of device operation and applications," Proceedings of the IEEE, vol. 90, no. 6, pp. 1022-1031, Jun 2002.

[14] O. I. Saadat, in Self-Aligned AlGaN/GaN Transistors for Sub-mm Wave Applications. (Master's thesis). Massachusetts Institute of Technology, 2010.

[15] M. Asif Khan, A. Bhattarai, J. N. Kuznia, and D. T. Olson, "High electron mobility transistor based on a GaNAlxGa1xN heterojunction," Applied Physics Letters, vol. 63, no. 9, pp. 1214$1215,1993$.

[16] P. D. Ye, B. Yang, K. K. Ng, J. Bude, G. D. Wilk, S. Halder, and J. C. M. Hwang, "GaN metaloxide-semiconductor high-electron-mobility-transistor with atomic layer deposited $\mathrm{A} 12 \mathrm{O} 3$ as gate dielectric," Applied Physics Letters, vol. 86, no. 6, 2005.

[17] J. Barth, C. Dyer, and E. Stassinopoulos, "Space, atmospheric, and terrestrial radiation environments," Nuclear Science, IEEE Transactions on, vol. 50, no. 3, pp. 466-482, June 2003.

[18] J. L. Barth, "Applying computer simulation tools to radiation effects problems," NSREC Short Course, July 1997.

[19] E. Petersen, in Single Event Effects in Aerospace. IEEE Press, John Wiley and Sons, Inc., 2011, pp. 13-72.

[20] J. H. Adams, A. F. Barghouty, M. H. Mendenhall, R. A. Reed, B. D. Sierawski, K. M. Warren, J. W. Watts, and R. A. Weller, "CRÉME: The 2011 revision of the cosmic ray effects on microelectronics code," IEEE Trans. Nucl. Sci., vol. 59, no. 6, pp. 3141-3147, Dec 2012.

[21] Space Environment (Natural and Artificial) - Galactic Cosmic Ray Model. ISO 15390, 2004.

[22] J. Wallmark and S. M. Marcus, "Minimum size and maximum packing density of nonredundant semiconductor devices," Proceedings of the IRE, vol. 50, no. 3, pp. 286-298, March 1962.

[23] M. Savage, J. Titus, T. Turflinger, R. Pease, and C. Poivey, "A comprehensive analog singleevent transient analysis methodology," Nuclear Science, IEEE Transactions on, vol. 51, no. 6, pp. 3546-3552, Dec 2004.

[24] J. Grant, W. Cunningham, A. Blue, V. OShea, J. Vaitkus, E. Gaubas, and M. Rahman, "Wide bandgap semiconductor detectors for harsh radiation environments," Nucl. Instr. Meth. A, vol. 546, no. 12, pp. $213-217,2005$.

[25] R. A. Reed, "Fundamental mechanisms for single particle-induced soft-errors," NSREC Short Course, July 2008.

[26] F. W. Sexton, "Destructive single-event effects in semiconductor devices and ICs," IEEE Trans. Nucl. Sci., vol. 50, no. 3, pp. 603-621, 2003.

[27] C. Wheatley, J. Titus, and D. Burton, "Single-event gate rupture in vertical power MOSFETs; an original empirical expression," Nuclear Science, IEEE Transactions on, vol. 41, no. 6, pp. 2152-2159, Dec 1994. 
[28] M. Allenspach, J. Brews, I. Mouret, R. Schrimpf, and K. Galloway, "Evaluation of SEGR threshold in power MOSFETs," Nuclear Science, IEEE Transactions on, vol. 41, no. 6, pp. 2160-2166, Dec 1994.

[29] P. McNulty, W. J. Beauvais, R. Reed, D. R. Roth, E. Stassinopoulos, and G. Brucker, "Charge collection at large angles of incidence [CMOS SRAM]," Nuclear Science, IEEE Transactions on, vol. 39, no. 6, pp. 1622-1629, Dec 1992.

[30] D. McMorrow, T. R. Weatherford, S. Buchner, A. R. Knudson, J. S. Melinger, L. H. Tran, and A. B. Campbell, "Single-event phenomena in GaAs devices and circuits," IEEE Trans. Nucl. Sci., vol. 43, no. 2, pp. 628-644, 1996.

[31] M. A. Hopkins and J. R. Srour, "Charge collection measurements on GaAs devices fabricated on semi-insulating substrates," IEEE Trans. Nucl. Sci., vol. 31, no. 6, pp. 1116-1120, 1984.

[32] T. R. Oldham, F. B. McLean, and J. M. Hartman, "Revised funnel calculations for heavy particles with high dE/dx," IEEE Trans. Nucl. Sci., vol. 33, no. 6, pp. 1646-1650, 1986.

[33] M. R. Brozel and G. E. Stillman, Eds., Properties of Gallium Arsenide, 3rd ed.

[34] D. McMorrow, J. B. Boos, A. R. Knudson, W. T. Lotshaw, D. Park, J. S. Melinger, B. R. Bennett, A. Torres, V. Ferlet-Cavrois, J.-E. Sauvestre, C. D'hose, and O. Flament, "Transient response of III-V field-effect transistors to heavy-ion irradiation," IEEE Trans. Nucl. Sci., vol. 51, no. 6, pp. 3324-3331, 2004.

[35] R. Zuleeg, J. K. Notthoff, and D. K. Nichols, "SEU of complementary gaas static rams due to heavy ions," IEEE Trans. Nucl. Sci., vol. 31, no. 6, pp. 1121-1123, 1984.

[36] V. Ramachandran, R. A. Reed, R. D. Schrimpf, D. McMorrow, J. B. Boos, M. P. King, E. X. Zhang, G. Vizkelethy, X. Shen, and S. T. Pantelides, "Single-event transient sensitivity of InAlSb/InAs/AlGaSb high electron mobility transistors," IEEE Trans. Nucl. Sci., vol. 59, no. 6, pp. 2691-2696, 2012.

[37] J. Wang, T. Zhao, J. Li, A. Q. Huang, R. Callanan, F. Husna, and A. Agarwal, "Characterization, modeling, and application of 10-kV SiC MOSFET," IEEE Trans. Elec. Dev., vol. 55, no. 8, pp. 1798-1806, 2008.

[38] M. Moll, "Radiation tolerant semiconductor sensors for tracking detectors," Nucl. Inst. Meth., vol. 565, no. 1, pp. $202-211,2006$.

[39] S. Onoda, T. Makino, S. Ono, S. Katakami, M. Arai, and T. Ohshima, "Spatial, LET and Range Dependence of Enhanced Charge Collection by Single Ion Strike in 4H-SiC MESFETs," IEEE Trans. Nucl. Sci., vol. 59, no. 4, pp. 742-748, 2012.

[40] H. Asai, K. Sugimoto, I. Nashiyama, Y. Iide, K. Shiba, M. Matsuda, and Y. Miyazaki, "Terrestrial neutron-induced single-event burnout in SiC power diodes," IEEE Trans. Nucl. Sci., vol. 59, no. 4, pp. 880-885, 2012.

[41] D. McMorrow, J. B. Boos, D. Park, S. Buchner, A. R. Knudson, and J. S. Melinger, "Chargecollection dynamics of InP-based high electron mobility transistors (HEMTs)," in Proc. 6th European Conference on Radiation and Its Effects on Components and Systems., 2001, pp. $132-137$. 
[42] J. A. Pellish, R. A. Reed, D. McMorrow, G. Vizkelethy, V. F. Cavrois, J. Baggio, P. Paillet, O. Duhamel, K. A. Moen, S. D. Phillips, R. M. Diestelhorst, J. D. Cressler, A. K. Sutton, A. Raman, M. Turowski, P. E. Dodd, M. L. Alles, R. D. Schrimpf, P. W. Marshall, and K. A. LaBel, "Heavy ion microbeam- and broadbeam-induced transients in SiGe HBTs," IEEE Trans. Nucl. Sci., vol. 56, no. 6, pp. 3078-3084, 2009.

[43] J. A. Pellish, R. A. Reed, D. McMorrow, J. S. Melinger, P. Jenkins, A. K. Sutton, R. M. Diestelhorst, S. D. Phillips, J. D. Cressler, V. Pouget, N. D. Pate, J. A. Kozub, M. H. Mendenhall, R. A. Weller, R. D. Schrimpf, P. W. Marshall, A. D. Tipton, and G. Niu, "Laser-induced current transients in silicon-germanium HBTs," IEEE Trans. Nucl. Sci., vol. 55, no. 6, pp. 2936-2942, 2008.

[44] E. X. Zhang, D. M. Fleetwood, N. D. Pate, R. A. Reed, A. F. Witulski, and R. D. Schrimpf, "Time-domain reflectometry measurements of total-ionizing-dose degradation of $n$ MOSFETs," IEEE Trans. Nucl. Sci., vol. 60, no. 6, pp. 4470-4475, Dec 2013.

[45] R. A. Weller, M. H. Mendenhall, R. A. Reed, R. D. Schrimpf, K. M. Warren, B. D. Sierawski, and L. W. Massengill, "Monte Carlo simulation of single event effects," IEEE Trans. Nucl. Sci., vol. 57, no. 4, pp. 1726-1746, 2010.

[46] A. Campbell, A. Knudson, D. McMorrow, W. Anderson, J. Roussos, S. Espy, S. Buchner, K. Kang, D. Kerns, and S. Kerns, "Ion induced charge collection in GaAs MESFETs," IEEE Trans. Nucl. Sci., vol. 36, no. 6, pp. 2292-2299, 1989.

[47] M. Breese, E. Vittone, G. Vizkelethy, and P. Sellin, "A review of ion beam induced charge microscopy," Nucl. Instr. Meth. B, vol. 264, no. 2, pp. 345 - 360, 2007.

[48] Synopsys Inc., "SDevice 2-D and 3-D device simulator."

[49] J. L. Titus, "An updated perspective of single event gate rupture and single event burnout in power MOSFETs," IEEE Trans. Nucl. Sci., vol. 60, no. 3, pp. 1912-1928, 2013. 